\title{
ON THE EXISTENCE OF SMOOTH DENSITIES FOR JUMP PROCESSES
}

\author{
Jean Picard \\ Laboratoire de Mathématiques Appliquées \\ URA $1501 \mathrm{du}$ CNRS \\ Université Blaise Pascal \\ 63177 Aubière Cedex, France \\ E-mail: picard@ucfma.univ-bpclermont.fr
}

\begin{abstract}
We consider a Lévy process $X_{t}$ and the solution $Y_{t}$ of a stochastic differential equation driven by $X_{t}$; we suppose that $X_{t}$ has infinitely many small jumps, but its Lévy measure may be very singular (for instance it may have a countable support). We obtain sufficient conditions ensuring the existence of a smooth density for $Y_{t}$; these conditions are similar to those of the classical Malliavin calculus for continuous diffusions. More generally, we study the smoothness of the law of variables $F$ defined on a Poisson probability space; the basic tool is a duality formula from which we estimate the characteristic function of $F$.
\end{abstract}

Mathematics Subject Classification (1991). 60H07 60J75 60J30

\section{Introduction}

Suppose that we are given a continuous diffusion process $Y_{t}$; it can be represented as a functional of a Wiener process. The aim of Malliavin's calculus introduced in [8] is to prove, by means of probabilistic methods, the existence of a smooth density for $Y_{t}$. The basic tool is an integration by parts formula on the Wiener space which enables to prove, under some conditions on the diffusion, that for any smooth function $g$,

$$
\left|\mathbb{E}\left[g^{\prime}\left(Y_{t}\right)\right]\right| \leq C \sup _{y}|g(y)|
$$

for a $C$ which does not depend on $g$; this implies that the law of $Y_{t}$ is absolutely continuous. Moreover, by iteration, the integration by parts formula shows that expectations of further derivatives of $g$ are also dominated by the supremum of $g$, and the following basic result enables to conclude about the existence of a smooth density.

Proposition 0.1. Let $F$ be a $\mathbb{R}^{d}$ valued variable; suppose that for any real-valued $C_{b}^{\infty}$ function $g$ defined on $\mathbb{R}^{d}$ and any $k \in \mathbb{N}^{d}$,

$$
\left|\mathbb{E}\left[\frac{\partial^{|k|} g}{\partial y_{1}^{k_{1}} \ldots \partial y_{d}^{k_{d}}}(F)\right]\right| \leq C_{k} \sup _{y}|g(y)|
$$

for a $C_{k}$ which does not depend on $g$. Then the law of $F$ has a $C_{b}^{\infty}$ density.

By choosing $g(y)=\exp (i w . y)$ for $w \in \mathbb{R}^{d}$, Proposition 0.1 is a consequence of the following result which is proved by means of the inversion formula for the Fourier transform. 
Proposition 0.2. Let $F$ be a $\mathbb{R}^{d}$ valued variable with characteristic function $\phi$, and let $k$ be a non-negative integer; if

$$
\int|w|^{k}|\phi(w)| d w<\infty
$$

then the law of $F$ has a $C_{b}^{k}$ density.

It should be noticed that the integration by parts formula on the Wiener space is obtained by applying small perturbations on the Wiener process in the direction of the Cameron-Martin space, so that these perturbations involve absolutely continuous changes of probability.

Now consider the case where $Y_{t}$ is a diffusion with jumps; more precisely, let $\mu$ be a measure on $\mathbb{R}^{m}$ which integrates the function $|x|^{2} \wedge 1$, let $\lambda^{+}(d t, d x), t \in \mathbb{R}_{+}, x \in \mathbb{R}^{m}$, be a space-time Poisson measure with intensity measure $\lambda^{-}(d t, d x)=d t \mu(d x)$, let $\lambda=\lambda^{+}-\lambda^{-}$, let $\chi \in \mathbb{R}^{m}$, and consider the Lévy process

$$
X_{t}=\chi t+\int_{0}^{t} \int_{\{|x| \leq 1\}} x d \lambda(s, x)+\int_{0}^{t} \int_{\{|x|>1\}} x d \lambda^{+}(s, x)
$$

without Brownian part and with Lévy measure $\mu$. We suppose that $Y_{t}$ is the solution of a stochastic differential equation (SDE) driven by $X_{t}$. The analogue of Malliavin's calculus for this framework was introduced in [2], and many papers were devoted to this subject afterwards. In order to prove an integration by parts formula in this case, one has to choose the type of small perturbations which should act on the Poisson measure $\lambda^{+}$; in the literature, these perturbations consist in moving the points of the support of $\lambda^{+}$, either in the time, or in the space directions; in both cases, assumptions ensuring the existence of a smooth density can be obtained for some functionals of $\lambda^{+}$. However, these results suffer some limitation. The technique of moving in the time direction (see [3]) has the advantage of being applicable without small jumps, for instance when $X_{t}$ is a standard Poisson process; it can be used to study some diffusions $Y_{t}$, but not $X_{t}$ itself (this is not surprising since the standard Poisson process is integer-valued). On the other hand, since the move should be small and should induce an absolutely continuous change of law, the technique of moving in the space directions considered in [2], [1], [6], [7] imposes regularity conditions on the measure $\mu$, which exclude for instance the case where the measure has a countable support. The aim of this work is to derive the existence of a smooth density for $Y_{t}$ in a framework which makes possible countably supported measures $\mu$; we will first obtain a result for a general class of functionals $F$ of the Poisson measure $\lambda^{+}$, then will apply it to the case $F=Y_{t}$.

For instance, let us consider the particular case where $F=X_{t}$ is the Lévy process itself; the absolute continuity of the law of $X_{t}$ was studied a long time ago (see [14], [13] and references therein), and it appears that the main problems concern the case where $\mu$ is singular. As it has been explained above, if for instance $\mu$ has a countable support, then the classical technique of Malliavin's calculus cannot be applied. Nevertheless, one must 
notice that the characteristic function of $X_{t}$ is given by the Lévy-Khintchine formula, so with Proposition 0.2 , one can obtain conditions on $\mu$ which are sufficient for the existence of a density and for its smoothness; these conditions state that $\mu$ should have enough mass near 0 , so that $X_{t}$ has many small jumps; then, even if $\mu$ is singular, the accumulation of small jumps forces $X_{t}$ to have a smooth density. In [2], it is explained how precise estimations on the tail of $\mu$ near the origin are equivalent to precise estimations of the characteristic function $\phi(w)$ as $w \rightarrow \infty$; here, we only need an upper bound for $\phi$; this will be done in $\S 1$.

Then we will consider more general functionals $F$ of a Poisson measure $\lambda^{+}$on a space $U$; our basic tool will be a duality formula on the Poisson space taken from [11] and [12] which will be the analogue of the integration by parts formula on the Wiener space (actually, as noticed in [10], [11], by means of the Fock space formalism, the two formulas can be transformed into each other). However, the analogue of the Wiener gradient operator is not any more a derivation, so the formula on the Poisson space cannot be used to estimate the expectation of $g^{\prime}(F)$ as on the Wiener space; the perturbation resulting from this operator indeed consists in adding a Dirac mass to $\lambda^{+}$. Nevertheless, it will appear that the duality formula can be used to estimate the characteristic function of $F$, and therefore, we will apply Proposition 0.2 rather than Proposition 0.1. Our assumptions will be of two types; the regularity assumption will say that adding masses to $\lambda^{+}$has smooth enough influence on $F$, and the non degeneracy assumption will say that this influence is large enough; the particular case where $F$ is a linear functional of $\lambda^{+}$will provide in $\S 1$ a second study of infinitely divisible laws; the general non linear case will be worked out in $\S 2$.

In $\S 3$, we will consider the case where $F$ is a functional of a finite-dimensional Lévy process $X_{t}$ with many small jumps, and in $\S 4$, the particular case where $F=Y_{t}$ is the solution of a SDE driven by $X_{t}$ will be dealt with. In classical Malliavin's calculus for continuous diffusions, the assumptions involve the vector fields of the SDE; for example, the ellipticity of the diffusion matrix implies the non-degeneracy of the diffusion. However, in the case of diffusions with jumps, the problem is made more difficult by strong geometrical interaction between the Lévy measure of $X$ and the equation. In this work, we will limit ourselves to the case where the SDE is driven by a Lévy process which has approximately the same number of small jumps in all the space directions. In this framework, the nondegeneracy assumption will be linked with the invertibility of an analogue of the Malliavin matrix, and a sufficient condition ensuring this invertibility and which can be easily read on the equation will be given; this condition consists of two parts; the first part describes the behaviour of the small jumps; it says that an analogue of the diffusion matrix is elliptic (more general conditions of Hörmander's type will not be studied in full generality, but an example will be given); the second part, which does not appear in the case of continuous diffusions, is concerned with big jumps and says that the semi-flow generated by the equation is locally injective. Some examples and counterexamples will be discussed.

\section{Infinitely divisible laws}

We first set some notation. Let $(U, \mathcal{U})$ be a Lusin space and let $\lambda^{-}$be a $\sigma$-finite infinite diffuse measure on $U$; in particular, the measured space $\left(U, \mathcal{U}, \lambda^{-}\right)$is isomorphic to $\mathbb{R}$ with 
its Borel $\sigma$-field and its Lebesgue measure. Let $\Omega$ be the space of integer-valued measures $\omega$ on $U$ such that $\omega(\{u\}) \leq 1$ for any $u$, and $\omega(A)<\infty$ as soon as $\lambda^{-}(A)<\infty$; we consider on $\Omega$ the canonical random measure

$$
\lambda^{+}(\omega, A)=\omega(A)
$$

the $\sigma$-field $\mathcal{F}$ generated by the variables $\lambda^{+}(A), A \in \mathcal{U}$, and the probability $\mathbb{P}$ under which $\lambda^{+}$is a Poisson measure with intensity $\lambda^{-}$; this means that $\lambda^{+}(A)$ is a Poisson variable with mean $\lambda^{-}(A)$, and that the variables $\lambda^{+}\left(A_{j}\right)$ are independent as soon as the sets $A_{j}$ are disjoint. We will denote by $\lambda=\lambda^{+}-\lambda^{-}$the compensated Poisson measure.

Remark. We suppose that $\lambda^{-}$is diffuse because formulas are simpler in this case; however, this is not a restriction, since one can always replace $U$ by $U \times[0,1]$ and $\lambda^{-}$by its product with the Lebesgue measure.

On the other hand, let $h$ be a $\mathbb{R}^{d}$ valued measurable function defined on $U$ such that

$$
\int\left(|h(u)|^{2} \wedge 1\right) d \lambda^{-}(u)<\infty
$$

and let $\bar{\chi} \in \mathbb{R}^{d}$. We consider the variable

$$
F=\bar{\chi}+\int h(u) 1_{[0,1]}(|h(u)|) d \lambda(u)+\int h(u) 1_{(1, \infty)}(|h(u)|) d \lambda^{+}(u)
$$

where the first integral is a stochastic integral, whereas the second one is for each $\omega \in \Omega$ a finite sum. Then the law of $F$ is infinitely divisible and its Lévy measure $\mu$ is the image of $\lambda^{-}$by $h$.

Proposition 1.1. Suppose that there exists an $\alpha \in(0,2)$ and a $c>0$ such that for any $\rho \in(0,1)$ and any unit vector $v$,

$$
\int_{\{x ;|v \cdot x| \leq \rho\}}|v \cdot x|^{2} d \mu(x) \geq c \rho^{\alpha} .
$$

Then $F$ has a $C_{b}^{\infty}$ density.

Remark. The condition (1.2) can also be stated by saying that

$$
\int_{\{x ;|w \cdot x| \leq 1\}}|w \cdot x|^{2} d \mu(x) \geq c|w|^{2-\alpha}
$$

for any vector $w$ such that $|w| \geq 1$.

Proof. The characteristic function of $F$ is given by the Lévy-Khintchine formula

$$
\phi(w)=\exp \left(i w \cdot \bar{\chi}+\int\left(e^{i w \cdot x}-1-i w \cdot x 1_{[0,1]}(|x|)\right) d \mu(x)\right)
$$


for $w \in \mathbb{R}^{d}$, so

$$
\begin{aligned}
|\phi(w)| & =\exp -\int(1-\cos (w \cdot x)) d \mu(x) \\
& \leq \exp -c^{\prime} \int_{\{|w \cdot x| \leq 1\}}|w \cdot x|^{2} d \mu(x) \leq \exp -c^{\prime \prime}|w|^{2-\alpha}
\end{aligned}
$$

for $|w| \geq 1$, from (1.3). We can conclude from Proposition 0.2.

In particular, one easily verifies that the condition (1.2) is satisfied in the following cases.

Corollary 1.2. Define

$$
V(\rho)=\int_{\{|x| \leq \rho\}} x x^{\star} d \mu(x) .
$$

(a) If the family of symmetric matrices $\rho^{-\alpha} V(\rho), 0<\rho<1$, is uniformly elliptic for some $\alpha \in(0,2)$, then $F$ has a $C_{b}^{\infty}$ density.

(b) In particular, if the ratio between the largest and smallest eigenvalues of $V(\rho)$ is bounded as $\rho \rightarrow 0$, and if

$$
\liminf _{\rho \rightarrow 0} \rho^{-\alpha} \int_{\{|x| \leq \rho\}}|x|^{2} d \mu(x)>0
$$

for some $\alpha \in(0,2)$, then $F$ has a $C_{b}^{\infty}$ density.

These results for infinitely divisible laws can of course be expressed in terms of Lévy processes (processes with stationary and independent increments). Suppose that $U=$ $\mathbb{R}_{+} \times \mathbb{R}^{m}$ and that $\lambda^{-}$is the product of the Lebesgue measure on $\mathbb{R}_{+}$and of a measure $\mu$ on $\mathbb{R}^{m}$ integrating $\left(|x|^{2} \wedge 1\right)$. If one considers on $U$ the function

$$
h_{t}(s, x)=1_{[0, t]}(s) x,
$$

and if $X_{t}$ denotes the variable $F$ of (1.1) corresponding to $h=h_{t}$ and $\bar{\chi}=\chi t$ in dimension $d=m$, then $X_{t}$ is the Lévy process of (0.1), and the image of $\lambda^{-}$by $h_{t}$ is in this case equal to $t \mu$. Thus, if the above assumptions are satisfied for $\mu$, then $X_{t}$ has a $C_{b}^{\infty}$ density for any $t>0$.

Remark 1. The condition of Corollary 1.2 is sufficient but not necessary for the condition (1.2) of Proposition 1.1. For example, suppose that $X_{t}$ is the two-dimensional process which consists of the Cauchy process with Lévy measure $d x / x^{2}$ on $\mathbb{R}$, and of its quadratic variation. Then the Lévy measure $\mu$ of $X_{t}$ is given by

$$
\int_{\mathbb{R}^{2}} g d \mu=\int_{\mathbb{R}} g\left(x, x^{2}\right) \frac{d x}{x^{2}}
$$

The eigenvalues of $V(\rho)$ are of order $\rho$ and $\rho^{3}$, so the assumption of Corollary 1.2 is not satisfied; however, after some calculation, one can check that the condition of Proposition 
1.1 holds, so $X_{t}$ has a smooth density. Later, we will study the solutions of SDEs driven by Lévy processes $X_{t}$, and the assumptions about the Lévy measure $\mu$ of $X_{t}$ will be those of Corollary 1.2(b), so this example will be excluded; it seems indeed hard to handle nonlinearities under more general conditions, though, as shown in [6], some results can be obtained from classical Malliavin's calculus.

Remark 2. One can also find conditions under which $X_{t}$ has not a $C^{\infty}$ density, but a $C^{n(t)}$ density, where $n(t)$ is a non-decreasing function tending to $\infty$ as $t \rightarrow \infty$; then (see [2], [6], [1]) the solutions of "well-behaved" SDEs driven by $X_{t}$ satisfy the same property, so one can say that the Lévy process is slowly regularizing. However, the method which we will use for non-linear functionals is not well adapted to study this type of behaviour.

Remark 3. If the law of $F$ is rotation-invariant $\beta$-stable $(0<\beta<2)$ so that $d \mu(x)$ is proportional to $d x /|x|^{d+\beta}$, then $V(\rho)$ is proportional to $\rho^{2-\beta} I$. Thus our condition can be viewed as a comparison with $(2-\alpha)$-stable laws.

We are now going to describe another proof of Proposition 1.1 which is more complicated, but which will be extended to more general functionals $F$. To this end, we need some other notation taken from [12]. We consider the transformations $\varepsilon_{u}^{-}$and $\varepsilon_{u}^{+}$of $\Omega$ which consist in removing or adding a mass at point $u$; they are defined by

$$
\varepsilon_{u}^{-} \omega(A)=\omega\left(A \cap\{u\}^{c}\right), \quad \varepsilon_{u}^{+} \omega(A)=\varepsilon_{u}^{-} \omega(A)+1_{A}(u) .
$$

We will have to use compositions of transformations of this type; if $u_{1} \neq u_{2} \in U$ and if $\theta_{1}$, $\theta_{2} \in\{-,+\}$, then

$$
\varepsilon_{u_{1}}^{\theta_{1}} \circ \varepsilon_{u_{2}}^{\theta_{2}}=\varepsilon_{u_{2}}^{\theta_{2}} \circ \varepsilon_{u_{1}}^{\theta_{1}}, \quad \varepsilon_{u}^{\theta_{1}} \circ \varepsilon_{u}^{\theta_{2}}=\varepsilon_{u}^{\theta_{1}} .
$$

Note also that $\varepsilon_{u}^{ \pm} \omega=\omega$ for all $\omega$ and $\lambda^{ \pm}$almost all $u$, so, if $Z_{u}$ is a positive measurable process indexed by $u \in U$, then

$$
\int\left(Z_{u} \circ \varepsilon_{u}^{ \pm}\right) d \lambda^{ \pm}(u)=\int Z_{u} d \lambda^{ \pm}(u)
$$

After these easily checked properties, let us state the lemma which appears as the basic result for our Poisson stochastic calculus.

Lemma 1.3. If $Z_{u}$ is a positive measurable process such that

$$
Z_{u} \circ \varepsilon_{u}^{+}=Z_{u} \circ \varepsilon_{u}^{-}
$$

then

$$
\mathbb{E} \int Z_{u} d \lambda^{+}(u)=\mathbb{E} \int Z_{u} d \lambda^{-}(u)
$$

Sketch of the proof. One shows that the processes $Z$ satisfying (1.8) are the positive functions on $U \times \Omega$ which are measurable with respect to the $\sigma$-field generated by sets $A \times B$ with $A \in \mathcal{U}$ and

$$
B \in \sigma\left(\lambda^{+}\left(A^{\prime}\right) ; A^{\prime} \in \mathcal{U}, A^{\prime} \cap A=\emptyset\right)
$$


Thus it is sufficient to prove the result when $Z$ is the indicator of such a set, and this is not difficult because $B$ and $\lambda^{+}(A)$ are independent (details of the proof can be found in $[12])$.

In particular, if $Z$ is a $\lambda^{-} \otimes \mathbb{P}$ integrable (subsequently, we will only say integrable) process satisfying the condition (1.8), then the variables $\int Z_{u} d \lambda^{ \pm}(u)$ are integrable, and formula (1.9) still holds. On the other hand, if we remove the condition (1.8), then the processes $Z_{u} \circ \varepsilon_{u}^{ \pm}$still satisfy it, so for any positive process, properties (1.7) of the integrals can be completed with

$$
\mathbb{E} \int\left(Z_{u} \circ \varepsilon_{u}^{ \pm}\right) d \lambda^{\mp}(u)=\mathbb{E} \int Z_{u} d \lambda^{ \pm}(u)
$$

Now, for functionals $F$ defined on $\Omega$, we introduce the operator

$$
D_{u} F=F \circ \varepsilon_{u}^{+}-F
$$

(with a slight modification with respect to the definition of [12]). Since the image of $\mathbb{P}$ by $\varepsilon_{u}^{+}$is not absolutely continuous with respect to $\mathbb{P}$, the variable $D_{u} F$ is not well defined for $u$ fixed when $F$ is defined almost surely; however, it is defined $d \lambda^{-}(u) \otimes d \mathbb{P}$ almost everywhere. We also consider an operator $\delta$ which operates on integrable processes $Z_{u}$ and which is defined by

$$
\delta(Z)=\int\left(Z_{u} \circ \varepsilon_{u}^{-}\right) d \lambda(u)=\int\left(Z_{u} \circ \varepsilon_{u}^{-}\right) d \lambda^{+}(u)-\int Z_{u} d \lambda^{-}(u) .
$$

The following result can be found in [11] or [12] and is an easy consequence of (1.10) and (1.6).

Lemma 1.4. Let $Z$ be a complex-valued integrable process and let $G$ be a complex-valued bounded variable. Then

$$
\mathbb{E}[G \delta(Z)]=\mathbb{E} \int Z_{u} D_{u} G d \lambda^{-}(u)
$$

Remark. The operators $D$ and $\delta$ are closable in $L^{2}$, and it appears that in the chaotic representation of square-integrable functionals, $D$ and $\delta$ correspond respectively to the annihilation and creation operators (see [10], [11]); thus the lemma describes the duality between these two operators. When transposed to the Wiener space, $D$ and $\delta$ are respectively the Malliavin derivative and the Skorohod integral; however, here, $D$ is not a derivation; it satisfies

$$
D_{u}\left(F_{1} F_{2}\right)=F_{1} D_{u} F_{2}+F_{2} D_{u} F_{1}+D_{u} F_{1} D_{u} F_{2}
$$

We now define non linear operators $\delta^{(n)}$ by induction on $n$ as follows; for $n=0$, put $\delta^{(0)}(Z)=1$; for $n \geq 0$, the domain of $\delta^{(n+1)}$ consists of processes $Z$ in the domain of $\delta^{(n)}$ such that $Z_{u} \delta^{(n)}(Z)$ is an integrable process, and for such a $Z$, we define

$$
\delta^{(n+1)}(Z)=\delta\left(Z \delta^{(n)}(Z)\right) .
$$


In particular, if $Z$ is deterministic and integrable, then it is not difficult to verify that

$$
\delta^{(n)}(Z)=\int_{S_{n}} Z_{u_{1}} \ldots Z_{u_{n}} d \lambda\left(u_{1}\right) \ldots d \lambda\left(u_{n}\right)
$$

with

$$
S_{n}=\left\{\left(u_{1}, \ldots, u_{n}\right) \in U^{n} ; \forall(i, j) \quad i \neq j \Rightarrow u_{i} \neq u_{j}\right\},
$$

so that $\delta^{(n)}(Z)$ is in the $n$th Poisson chaos; if $U$ is identified with an interval of $\mathbb{R}$,

$$
\delta^{(n)}(Z)=n ! \int_{\left\{u_{1}<\ldots<u_{n}\right\}} Z_{u_{1}} \ldots Z_{u_{n}} d \lambda\left(u_{1}\right) \ldots d \lambda\left(u_{n}\right) .
$$

Lemma 1.5. Let $Z$ be an integrable process and let $G$ be a bounded variable such that

$$
G=\int Z_{u} D_{u} G d \lambda^{-}(u)
$$

If $Z$ is in the domain of $\delta^{(n)}$, then

$$
\mathbb{E}\left[G \delta^{(n)}(Z)\right]=\mathbb{E}[G] .
$$

Proof. This is evident for $n=0$ and

$$
\begin{aligned}
\mathbb{E}\left[G \delta^{(n+1)}(Z)\right] & =\mathbb{E}\left[G \delta\left(Z \delta^{(n)}(Z)\right)\right] \\
& =\mathbb{E}\left[\delta^{(n)}(Z) \int Z_{u} D_{u} G d \lambda^{-}(u)\right] \\
& =\mathbb{E}\left[G \delta^{(n)}(Z)\right]
\end{aligned}
$$

from the duality formula (1.12) and the assumption (1.15).

Second proof of Proposition 1.1. The assumption (1.3) can be written in the form

$$
\int_{A}|h(u) \cdot w|^{2} d \lambda^{-}(u) \geq c|w|^{2-\alpha}
$$

for $|w| \geq 1$ and

$$
A=A(w)=\{u \in U ;|h(u) . w| \leq 1\} .
$$

Thus there exists a $\rho(w)>0$ such that

$$
\int_{B}|h(u) \cdot w|^{2} d \lambda^{-}(u) \geq c|w|^{2-\alpha} / 2
$$

with

$$
B=B(w)=\{u \in A(w) ;|h(u)| \geq \rho(w)\} .
$$


Note that $B$ has $\lambda^{-}$-finite measure. Then

$$
\begin{aligned}
\int_{B}\left|e^{i w \cdot h(u)}-1\right|^{2} d \lambda^{-}(u) & \geq c^{\prime} \int_{B}|w \cdot h(u)|^{2} d \lambda^{-}(u) \\
& \geq c c^{\prime}|w|^{2-\alpha} / 2 .
\end{aligned}
$$

Now consider the deterministic function

$$
Z_{u}=1_{B}(u)\left(e^{-i w \cdot h(u)}-1\right) / \int_{B}\left|e^{i w \cdot h(u)}-1\right|^{2} d \lambda^{-}(u) .
$$

and the variable $G=G(w)=\exp (i w \cdot F)$. Then $Z$ is bounded, and is zero outside $B$ which has finite measure, so $Z$ is integrable; moreover

$$
D_{u} G=\left(e^{i w \cdot h(u)}-1\right) G
$$

for $\lambda^{-}$-almost every $u$, so the assumption of Lemma 1.5 is satisfied, and therefore the characteristic function $\phi$ of $F$ satisfies

$$
|\phi(w)|=|\mathbb{E}[G]|=\left|\mathbb{E}\left[G \delta^{(n)}(Z)\right]\right| \leq \mathbb{E}\left[\left|\delta^{(n)}(Z)\right|^{2}\right]^{1 / 2}
$$

for any $n$. But from (1.14),

$$
\begin{aligned}
\mathbb{E}\left[\left|\delta^{(n)}(Z)\right|^{2}\right] & =n !\left(\int\left|Z_{u}\right|^{2} d \lambda^{-}(u)\right)^{n} \\
& =n !\left(\int_{B}\left|e^{i w \cdot h(u)}-1\right|^{2} d \lambda^{-}(u)\right)^{-n} \\
& \leq C_{n}|w|^{n(\alpha-2)} .
\end{aligned}
$$

Thus, for any $k \geq 0$,

$$
|w|^{k}|\phi(w)| \leq C_{n}|w|^{k-n(2-\alpha) / 2}
$$

is proved to be integrable by choosing a large enough $n$.

\section{The main result}

We now want to prove a result for non linear functionals $F$. The basic idea has been developed in last proof, but it involves here more technicalities. We use the notation of $\S 1$; in particular, $\varepsilon_{u}^{+}$and $D_{u}$ are respectively defined in (1.5) and (1.11); if $\tau=\left(u_{1}, \ldots, u_{k}\right) \in$ $U^{k}$, we consider the transformation

$$
\varepsilon_{\tau}^{+}=\varepsilon_{u_{1}}^{+} \circ \ldots \circ \varepsilon_{u_{k}}^{+}
$$

and the operator

$$
D_{\tau}=D_{u_{1}} \ldots D_{u_{k}}
$$


If $\gamma$ is a real function defined on $U$, we also extend $\gamma$ to $U^{k}$ by putting

$$
\gamma(\tau)=\gamma\left(u_{1}\right) \ldots \gamma\left(u_{k}\right)
$$

In the case $k=0$, we use the convention $\varepsilon_{\emptyset}^{+} \omega=\omega, D_{\emptyset} F=F$ and $\gamma(\emptyset)=1$. The measure $\lambda$ is extended to $U^{k}$ by putting

$$
d \lambda\left(u_{1}, \ldots, u_{k}\right)=d \lambda\left(u_{1}\right) \ldots d \lambda\left(u_{k}\right)
$$

and a similar convention is adopted for $\lambda^{ \pm}$and $|\lambda|=\lambda^{+}+\lambda^{-}$. In particular, the expression "almost everywhere" on $U^{k}$ will refer to the extension of $\lambda^{-}$. The positive constant numbers will be denoted by $c$ or $C$, and may vary from line to line; if they depend on some parameter, this is emphasized by an index.

Theorem 2.1. Let $\gamma$ be a positive function defined on $U$ such that $\gamma \wedge 1$ is in $L^{2}\left(U, \lambda^{-}\right)$; for $0<\rho \leq 1$, define

$$
A(\rho)=\{u \in U ; \gamma(u) \leq \rho\}
$$

and suppose that

$$
\int_{A(\rho)} \gamma(u)^{2} d \lambda^{-}(u) \geq c \rho^{\alpha}
$$

for some $\alpha \in(0,2)$. Let $F$ be a real-valued variable such that

(a) for any $p \in(1, \infty)$, any $k \geq 1$ and almost any $\tau \in A(1)^{k}$, one has

$$
\left\|D_{\tau} F\right\|_{p} \leq C_{p, k} \gamma(\tau)
$$

(b) there exists some $\alpha / 2<\beta \leq 1$ such that for any $p \in(1, \infty)$, any $\rho \in(0,1)$, any $k \geq 1$ and almost any $\tau \in A(\rho)^{k}$, one has

$$
\left\|\left(\int_{A(\rho)}\left|D_{u} F\right|^{2} 1_{\left\{\left|D_{u} F\right| \leq \rho^{\beta}\right\}} d \lambda^{-}(u)\right)^{-1} \circ \varepsilon_{\tau}^{+}\right\|_{p} \leq C_{p, k}\left(\int_{A(\rho)} \gamma(u)^{2} d \lambda^{-}(u)\right)^{-1} .
$$

Then $F$ has a $C_{b}^{\infty}$ density.

Assumption (a) is a regularity assumption on $F$ with respect to the perturbations $\varepsilon_{\tau}^{+}$; it means that each iteration of a $D_{u}$ multiplies the order of magnitude by $\gamma(u)$; in $\S 3$, we will take $U=[0, T] \times \mathbb{R}^{m}$ and $\gamma(t, x)=|x|$, so, for $k=1$, (a) will mean that a jump of size $x$ at time $t$ of the process $X_{t}$ of (0.1) should induce on $F$ a perturbation of order $|x|$. On the other hand, (b) is a non-degeneracy assumption; it says that $\left|D_{u} F\right|$ is bounded below by $\gamma(u)$ in some sense; actually, an application of the Jensen inequality proves that $\left\|H^{-1}\right\|_{p} \geq\|H\|_{p}^{-1}$, and one can deduce from (2.2) that the right-hand side of (2.3) is dominated by the left-hand side; thus condition (b) means that the two sides are equivalent when $\rho$ tends to 0 . Let us now state the extension of Theorem 2.1 to the case of vector-valued variables $F$. 
Theorem 2.2. Let $F$ be a $\mathbb{R}^{d}$ valued variable; suppose that for any unit vector $v \in S^{d-1}$, there exists a function $\gamma_{v}$ such that the variable F.v satisfies the assumptions of Theorem 2.1 with $\gamma=\gamma_{v}$. If the constant numbers $\alpha, \beta, c$ and $C_{p, k}$ can be chosen independently of $v$, then $F$ has a $C_{b}^{\infty}$ density.

Example. For variables defined by (1.1), one has $D_{u} F=h(u)$, so iterates of the operators $D_{u_{i}}$ are zero on $F$. Thus (2.2) and (2.3) are satisfied with $\gamma_{v}(u)=|h(u) \cdot v|$ and $\beta=1$, so Theorem 2.2 reduces in this case to Proposition 1.1.

Remark. The theorems also hold when $F$ is defined on the product of $\Omega$ with another probability space; this other space may for instance be a Wiener space or another Poisson space.

Theorem 2.1 will be proved by estimating the characteristic function $\phi(w)$ of $F$ as $w \rightarrow \infty$; more precisely, we will check that for any $n$, the function $|w|^{-n} \phi(w)$ is bounded for $|w| \geq 1$ by some number depending only on $n, \alpha, \beta, c$ and $C_{p, k}$; then we will deduce that $F$ has a smooth density as in $\S 1$. On the other hand, if $F$ is vector-valued, we can apply this estimation to the variables $F . v$ for $v$ a unit vector; this implies that $w^{-n} \phi(w v)$ is bounded uniformly in $w \geq 1, v \in S^{d-1}$; we deduce that $|w|^{-n} \phi(w)$ is bounded uniformly in $w \in \mathbb{R}^{d},|w| \geq 1$, so that Theorem 2.2 actually follows from Theorem 2.1. Thus the remainder of this section is devoted to the proof of Theorem 2.1 for a real-valued $F$.

For $w \in \mathbb{R},|w| \geq 1$, and $\zeta \geq 0$, consider

$$
B(w, \zeta)=\left\{u \in U ; \quad \zeta \leq \gamma(u) \leq|w|^{-1 / \beta}\right\} .
$$

By taking $\rho=|w|^{-1 / \beta}$, the assumptions (2.1) and (2.3) are written in the form

$$
\begin{gathered}
\int_{B} \gamma(u)^{2} d \lambda^{-}(u) \geq c|w|^{-\alpha / \beta} \\
\left\|\left(\int_{B}\left|D_{u} F\right|^{2} 1_{\left\{\left|D_{u} F\right| \leq 1 /|w|\right\}} d \lambda^{-}(u)\right)^{-1} \circ \varepsilon_{\tau}^{+}\right\|_{p} \leq C_{p, k}\left(\int_{B} \gamma(u)^{2} d \lambda^{-}(u)\right)^{-1}
\end{gathered}
$$

for $\tau \in B^{k}$ and $B=B(w, 0)$. This implies that there exists $\zeta_{0}=\zeta_{0}(w, p, k)>0$ such that (2.5) and (2.6) are also satisfied for $B=B(w, \zeta), 0 \leq \zeta \leq \zeta_{0}$, and after a modification of $c$ and $C_{p, k}$; the sets $B(w, \zeta), \zeta>0$, have the advantage of having $\lambda^{-}$finite measure. Now consider the process

$$
Z_{u}=1_{B}(u)\left(e^{-i w D_{u} F}-1\right) / \int_{B}\left|e^{i w D_{v} F}-1\right|^{2} d \lambda^{-}(v)
$$

for $B=B(w, \zeta)$. Then $G=e^{i w F}$ satisfies

$$
D_{u} G=\left(e^{i w D_{u} F}-1\right) G,
$$

so (1.15) is satisfied, and from Lemma 1.5, the estimation of $\mathbb{E}[G]$ can be reduced to the proof of $Z \in \operatorname{Dom}\left(\delta^{(n)}\right)$ for $\zeta>0$ small enough, and to the estimation of $\delta^{(n)}(Z)$. Recall that $S_{n}$ is the subset of $U^{n}$ consisting of vectors with distinct components. 
Lemma 2.3. Let $Y_{\tau}$ be a positive process indexed by $\tau=\left(u_{1}, \ldots, u_{n}\right) \in U^{n}$; consider $\left(\theta_{1}, \ldots, \theta_{n}\right) \in\{-,+\}^{n}$. Then

$$
\begin{aligned}
& \mathbb{E} \int_{S_{n}} Y_{\tau} d \lambda^{\theta_{1}}\left(u_{1}\right) \ldots d \lambda^{\theta_{n}}\left(u_{n}\right) \\
& =\mathbb{E} \int_{U^{n}}\left(Y_{\tau} \circ \varepsilon_{u_{1}}^{\theta_{1}} \circ \ldots \circ \varepsilon_{u_{n}}^{\theta_{n}}\right) d \lambda^{-}\left(u_{1}\right) \ldots d \lambda^{-}\left(u_{n}\right) .
\end{aligned}
$$

Remark. In the right-hand side, the $\varepsilon_{u_{j}}^{-}$corresponding to $\theta_{j}=-$ can be omitted.

Proof. From Fubini's theorem and (1.10), the left-hand side is equal to

$$
\begin{aligned}
& \mathbb{E} \int_{U}\left(\int_{S_{n-1}} 1_{S_{n}}(\tau) Y_{\tau} d \lambda^{\theta_{2}}\left(u_{2}\right) \ldots d \lambda^{\theta_{n}}\left(u_{n}\right)\right) d \lambda^{\theta_{1}}\left(u_{1}\right) \\
& =\mathbb{E} \int_{U}\left(\int_{S_{n-1}} 1_{S_{n}}(\tau) Y_{\tau} d \lambda^{\theta_{2}}\left(u_{2}\right) \ldots d \lambda^{\theta_{n}}\left(u_{n}\right)\right) \circ \varepsilon_{u_{1}}^{\theta_{1}} d \lambda^{-}\left(u_{1}\right) \\
& =\int_{U}\left(\mathbb{E} \int_{S_{n-1}}\left(Y_{\tau} \circ \varepsilon_{u_{1}}^{\theta_{1}}\right) d \lambda^{\theta_{2}}\left(u_{2}\right) \ldots d \lambda^{\theta_{n}}\left(u_{n}\right)\right) d \lambda^{-}\left(u_{1}\right) .
\end{aligned}
$$

It is then not difficult to prove the lemma by induction on $n$.

Lemma 2.4. Let $Y_{\tau}$ be a process indexed by $\tau=\left(\tau_{1}, \tau_{2}\right) \in U^{k} \times U^{l}$. If

$$
\mathbb{E} \int\left|Y_{\tau}\right|\left|d \lambda\left(\tau_{1}\right)\right| d \lambda^{+}\left(\tau_{2}\right)<\infty
$$

then

$$
\mathbb{E} \int_{S_{k+l}} Y_{\tau} d \lambda\left(\tau_{1}\right) d \lambda^{+}\left(\tau_{2}\right)=\mathbb{E} \int_{U^{k+l}} D_{\tau_{1}} Y_{\tau} \circ \varepsilon_{\tau_{2}}^{+} d \lambda^{-}(\tau) .
$$

Remark. When $\tau_{1}$ and $\tau_{2}$ consist of distinct components, then

$$
\left(D_{\tau_{1}} Y_{\tau}\right) \circ \varepsilon_{\tau_{2}}^{+}=D_{\tau_{1}}\left(Y_{\tau} \circ \varepsilon_{\tau_{2}}^{+}\right)
$$

Since this holds for almost any $\left(\tau_{1}, \tau_{2}\right)$, the right-hand side of the lemma is not ambiguous. Proof. It is sufficient to expand $d \lambda\left(\tau_{1}\right)$ and to apply previous lemma to each term.

Lemma 2.5. For any $n$, the process $Z$ defined by (2.7) is in the domain of $\delta^{(n)}$ for $\zeta>0$ small enough, and

$$
\delta^{(n)}(Z)=\int_{S_{n}} \prod_{j=1}^{n}\left(Z_{u_{j}} \circ \varepsilon_{u_{1}}^{-} \circ \ldots \circ \varepsilon_{u_{j}}^{-}\right) d \lambda\left(u_{1}\right) \ldots d \lambda\left(u_{n}\right)
$$


Proof. This result is again proved by induction on $n$. For $n=1$, we have to prove the integrability of $Z$; to this end, note that $Z_{u}$ is zero for $u$ outside $B=B(w, \zeta)$ which has $\lambda^{-}$finite measure, and that

$$
\left|Z_{u}\right| \leq C w^{-2}\left(\int_{B}\left|D_{v} F\right|^{2} 1_{\left\{\left|D_{v} F\right| \leq 1 /|w|\right\}} d \lambda^{-}(v)\right)^{-1}
$$

The right-hand side is integrable from (2.6) for $\zeta$ small enough, so $Z$ is integrable and (2.8) for $n=1$ is evident. Now suppose that the result holds at rank $n$; we have to prove the integrability of $Z_{u} \delta^{(n)}(Z)$; from the formula (2.8) written at rank $n$,

$$
\begin{aligned}
\left|\delta^{(n)}(Z)\right| & \leq \int_{S_{n}} \prod_{j=1}^{n}\left|Z_{u_{j}} \circ \varepsilon_{u_{1}}^{-} \circ \ldots \circ \varepsilon_{u_{j}}^{-}\right|\left|d \lambda\left(u_{1}\right)\right| \ldots\left|d \lambda\left(u_{n}\right)\right| \\
& =\sum_{\theta \in\{-,+\}} \int_{S_{n}} \prod_{j=1}^{n}\left|Z_{u_{j}} \circ \varepsilon_{u_{1}}^{-} \circ \ldots \circ \varepsilon_{u_{j}}^{-}\right| d \lambda^{\theta_{1}}\left(u_{1}\right) \ldots d \lambda^{\theta_{n}}\left(u_{n}\right) .
\end{aligned}
$$

Thus

$$
\begin{aligned}
& \mathbb{E} \int\left|Z_{u}\right|\left|\delta^{(n)}(Z)\right| d \lambda^{-}(u) \\
& \leq \sum_{\theta \in\{-,+\}^{n}} \mathbb{E} \int_{S_{n+1}}\left|Z_{u_{0}}\right| \prod_{j=1}^{n}\left|Z_{u_{j}} \circ \varepsilon_{u_{1}}^{-} \circ \ldots \circ \varepsilon_{u_{j}}^{-}\right| d \lambda^{\theta_{1}}\left(u_{1}\right) \ldots d \lambda^{\theta_{n}}\left(u_{n}\right) d \lambda^{-}\left(u_{0}\right) \\
& =\sum_{\theta \in\{-,+\}^{n}} \mathbb{E} \int_{U^{n+1}}\left|Z_{u_{0}} \circ \varepsilon_{U(\tau, 0, \theta)}^{+}\right| \prod_{j=1}^{n}\left|Z_{u_{j}} \circ \varepsilon_{U(\tau, j, \theta)}^{+}\right| d \lambda^{-}\left(u_{0}\right) \ldots d \lambda^{-}\left(u_{n}\right)
\end{aligned}
$$

from Lemma 2.3, where $\tau=\left(u_{0}, \ldots, u_{n}\right)$ and $U(\tau, j, \theta)$ consists of the components $u_{k}$ such that $j<k \leq n$ and $\theta_{k}=+$. On the other hand, one deduces from (2.9) and (2.6) that for any $p$, the variables $Z_{u} \circ \varepsilon_{\tau}^{+}$are bounded in $L^{p}$ for $\zeta$ small enough; since they are equal to 0 outside $B$, this implies that the above expression is finite. Thus $Z$ is in the domain of $\delta^{(n+1)}$ and

$$
\begin{aligned}
\delta^{(n+1)}(Z) & =\int\left(Z_{u_{0}} \circ \varepsilon_{u_{0}}^{-}\right) \delta^{(n)}(Z) \circ \varepsilon_{u_{0}}^{-} d \lambda\left(u_{0}\right) \\
& =\int\left(Z_{u_{0}} \circ \varepsilon_{u_{0}}^{-}\right)\left(\int_{S_{n}} \prod_{j=1}^{n}\left(Z_{u_{j}} \circ \varepsilon_{u_{0}}^{-} \circ \ldots \circ \varepsilon_{u_{j}}^{-}\right) 1_{\left\{u_{j} \neq u_{0}\right\}} d \lambda\left(u_{1}\right) \ldots d \lambda\left(u_{n}\right)\right) d \lambda\left(u_{0}\right)
\end{aligned}
$$

satisfies (2.8).

In order to compute the variance of $\delta^{(n)}(Z)$, we need a new multi-indexed process. For $\tau=\left(u_{1}, \ldots, u_{2 n}\right) \in U^{2 n}$, define

$$
\widehat{Z}(\tau)=1_{S_{n} \times S_{n}}(\tau) \prod_{j=1}^{n}\left(Z_{u_{j}} \circ \varepsilon_{u_{1}}^{-} \circ \ldots \circ \varepsilon_{u_{j}}^{-}\right) \prod_{j=n+1}^{2 n}\left(\bar{Z}_{u_{j}} \circ \varepsilon_{u_{n+1}}^{-} \circ \ldots \circ \varepsilon_{u_{j}}^{-}\right)
$$

where $\bar{Z}_{u}$ is the conjugate complex number of $Z_{u}$. Let $\widetilde{Z}(\tau)$ be the random function of $\tau$ obtained from $\widehat{Z}$ by symmetrization. 
Lemma 2.6. For any $n$,

$$
\mathbb{E}\left[\left|\delta^{(n)}(Z)\right|^{2}\right]=\sum_{k+l=n} \frac{(2 n) !}{2^{l}(2 k) ! l !} \mathbb{E} \int_{U^{2 k} \times U^{l}}\left(D_{\tau_{1}} \widetilde{Z}\left(\tau_{1}, \tau_{2}, \tau_{2}\right) \circ \varepsilon_{\tau_{2}}^{+}\right) d \lambda^{-}\left(\tau_{1}\right) d \lambda^{-}\left(\tau_{2}\right),
$$

where for each $(k, l)$, the process $\left(\tau_{1}, \tau_{2}\right) \mapsto D_{\tau_{1}} \widetilde{Z}\left(\tau_{1}, \tau_{2}, \tau_{2}\right) \circ \varepsilon_{\tau_{2}}^{+}$is $\left(\lambda^{-}\right)^{\otimes(2 k+l)} \otimes \mathbb{P}$ integrable for $\zeta$ small enough.

Proof. From Lemma 2.5, it is clear that

$$
\left|\delta^{(n)}(Z)\right|^{2}=\int_{U^{2 n}} \widehat{Z}(\tau) d \lambda(\tau)=\int_{U^{2 n}} \widetilde{Z}(\tau) d \lambda(\tau)
$$

In this integral, the components of $\tau$ are not supposed to be distinct, and equal components cannot be neglected in the integration with respect to $\lambda^{+}$; however, since $\widetilde{Z}(\tau)$ is zero as soon as three components of $\tau$ are equal, there only may be some pairs of components which are equal; when two components are equal, we can use

$$
d \lambda(u) d \lambda(u)=d \lambda^{+}(u)
$$

By developing on the possible numbers $l=0, \ldots, n$ of such pairs and by using the symmetry of $\widetilde{Z}$, we obtain

$$
\left|\delta^{(n)}(Z)\right|^{2}=\sum_{k+l=n} \frac{(2 n) !}{2^{l}(2 k) ! l !} \int_{S_{2 k+l}} \widetilde{Z}\left(\tau_{1}, \tau_{2}, \tau_{2}\right) d \lambda\left(\tau_{1}\right) d \lambda^{+}\left(\tau_{2}\right)
$$

with $\tau_{1}$ of length $2 k, \tau_{2}$ of length $l$, and where the coefficient is the number of sets of $l$ disjoint pairs in $\{1, \ldots, 2 n\}$. From the definition of $\widetilde{Z}$ and since the moments of $Z_{u} \circ \varepsilon_{\tau}^{+}$ are bounded, we prove that

$$
\mathbb{E} \int_{S_{2 k+l}}\left|\widetilde{Z}\left(\tau_{1}, \tau_{2}, \tau_{2}\right)\right|\left|d \lambda\left(\tau_{1}\right)\right| d \lambda^{+}\left(\tau_{2}\right)<\infty
$$

for $\zeta$ small enough, so we can apply Lemma 2.4 in order to conclude.

Thus we have to estimate the right-hand side of (2.11); to this end, we still need some technical results. If $H_{\tau}$ is indexed by $\tau \in \bigcup_{k} B^{k}$, the notation $H_{\tau}=O(\gamma(\tau))$ will mean that for any $k$, for almost any $\tau \in B^{k}$, and for any $p$, one has

$$
\left\|H_{\tau}\right\|_{p} \leq C_{p, k} \gamma(\tau)
$$

for $\zeta>0$ small enough. If $H$ depends on some other parameters, the constant $C_{p, k}$ must be uniform; in particular, $H$ will generally depend on $w$. 
Lemma 2.7. Let $H, H_{1}, H_{2}$ be complex variables which may depend on some parameters.

(i) If $D_{\tau} H=O(\gamma(\tau))$, then $D_{\tau_{1}} H \circ \varepsilon_{\tau_{2}}^{+}=O\left(\gamma\left(\tau_{1}\right)\right)$.

(ii) If $D_{\tau} H_{j}=O(\gamma(\tau))$ for $j=1,2$ and $\tau$ of length at most $k$, then $D_{\tau}\left(H_{1} H_{2}\right)=O(\gamma(\tau))$ for $\tau$ of length at most $k$.

(iii) If $D_{\tau} H=O(\gamma(\tau))$ and $H^{-1} \circ \varepsilon_{\tau}^{+}=O(1)$, then $D_{\tau}\left(H^{-1}\right)=O(\gamma(\tau))$.

Proof. The first result is proved by induction on the length of $\tau_{2}$; this is evident when the length is 0 ; suppose that it holds for any $\tau_{2}$ of length $k$, and let us prove it for $\tau_{2}^{\prime}=\left(\tau_{2}, v\right)$ of length $k+1$; to this end, note that

$$
D_{\tau_{1}} H \circ \varepsilon_{\left(\tau_{2}, v\right)}^{+}=D_{\tau_{1}} H \circ \varepsilon_{\tau_{2}}^{+}+D_{\left(\tau_{1}, v\right)} H \circ \varepsilon_{\tau_{2}}^{+}
$$

is of order $\gamma\left(\tau_{1}\right)+\gamma\left(\tau_{1}\right) \gamma(v)$ from the induction assumption; moreover $\gamma(v) \leq 1$ on $B$, so (i) is proved. The second result is also evident for $k=0$; suppose that it holds at rank $k$ and let us prove it for $\tau^{\prime}=(\tau, v)$ of length $k+1$; from (1.13), we have

$$
\begin{aligned}
D_{\tau^{\prime}}\left(H_{1} H_{2}\right) & =D_{\tau} D_{v}\left(H_{1} H_{2}\right)=D_{\tau}\left(D_{v} H_{1} D_{v} H_{2}+H_{1} D_{v} H_{2}+H_{2} D_{v} H_{1}\right) \\
& =\gamma(v)^{2} D_{\tau}\left(H_{1}^{\prime} H_{2}^{\prime}\right)+\gamma(v) D_{\tau}\left(H_{1} H_{2}^{\prime}\right)+\gamma(v) D_{\tau}\left(H_{1}^{\prime} H_{2}\right)
\end{aligned}
$$

with $H_{j}^{\prime}=D_{v} H_{j} / \gamma(v)$; from the induction assumption applied to the variables $H_{j}$ and $H_{j}^{\prime}$, we deduce that this expression is of order $\gamma(\tau) \gamma(v)=\gamma\left(\tau^{\prime}\right)$, so (ii) is proved. The third result is also proved by induction on the length of $\tau$. When the length is 1 , the result follows easily from the assumptions and

$$
D_{v}(1 / H)=-\frac{D_{v} H}{H\left(H \circ \varepsilon_{v}^{+}\right)}
$$

If the result holds for $\tau$ of length $k$ and in order to prove it for $\tau^{\prime}=(\tau, v)$ of length $k+1$, we apply the operator $D_{\tau}$ to $(2.12)$ and obtain

$$
\frac{1}{\gamma(v)} D_{\tau^{\prime}}(1 / H)=-D_{\tau}\left(\frac{D_{v} H}{\gamma(v)} H^{-1}\left(H \circ \varepsilon_{v}^{+}\right)^{-1}\right)
$$

From (ii), we only have to estimate $D_{\tau}$ applied to each of the three terms $D_{v} H / \gamma(v), H^{-1}$ and $\left(H \circ \varepsilon_{v}^{+}\right)^{-1}$. For the first one, we use the assumption; for the second one, we use the induction assumption; for the third one, we apply the induction assumption to the variable $H \circ \varepsilon_{v}^{+}$which satisfies

$$
D_{\tau}\left(H \circ \varepsilon_{v}^{+}\right)=O(\gamma(\tau))
$$

from (i). Thus all these terms are of order $\gamma(\tau)$, and we can conclude that $D_{\tau^{\prime}}(1 / H)$ is of order $\gamma(v) \gamma(\tau)=\gamma\left(\tau^{\prime}\right)$

Remark. Subsequently, we will consider variables or processes which are smooth in the sense that each application of $D_{u}$ multiplies their order of magnitude by $\gamma(u)$. Lemma 2.7 says that this class of smooth variables is stable by some operations. 
Lemma 2.8. For $|w| \geq 1$, almost any $u \in B$, almost any $\tau \in B^{k}$ and $\zeta$ small enough, one has

$$
\left\|D_{\tau} Z_{u}\right\|_{p} \leq C_{p, k} \gamma(u) \gamma(\tau)\left(|w| \int_{B} \gamma(v)^{2} d \lambda^{-}(v)\right)^{-1}
$$

Proof. We first prove by induction on the length $k$ of $\tau_{1}$ that

$$
D_{\tau_{1}}\left(\exp \left(-i w D_{\tau_{2}} F\right)-1\right)=O\left(|w| \gamma\left(\tau_{1}\right) \gamma\left(\tau_{2}\right)\right)
$$

for $\tau_{2}$ of length $l \geq 1$. For $k=0$, we have

$$
\left|\exp \left(-i w D_{\tau_{2}} F\right)-1\right| \leq|w|\left|D_{\tau_{2}} F\right|=O\left(|w| \gamma\left(\tau_{2}\right)\right)
$$

from assumption (2.2). Now suppose that the estimation holds at rank $k$, consider a vector $\tau_{1}$ of length $k$, and $\tau_{1}^{\prime}=\left(\tau_{1}, v\right)$ of length $k+1$. Then

$$
\begin{aligned}
& D_{v}\left(\exp \left(-i w D_{\tau_{2}} F\right)-1\right) \\
& \quad=\exp \left(-i w D_{\tau_{2}} F-i w D_{\tau_{2}^{\prime}} F\right)-\exp \left(-i w D_{\tau_{2}} F\right) \\
& \quad=\left(\exp \left(-i w D_{\tau_{2}} F\right)-1\right)\left(\exp \left(-i w D_{\tau_{2}^{\prime}} F\right)-1\right)+\left(\exp \left(-i w D_{\tau_{2}^{\prime}} F\right)-1\right)
\end{aligned}
$$

with $\tau_{2}^{\prime}=\left(\tau_{2}, v\right)$. We apply the operator $D_{\tau_{1}}$ and use Lemma 2.7(ii) and the induction assumption to check that the expression is dominated by

$$
\gamma\left(\tau_{1}\right)\left(w^{2} \gamma\left(\tau_{2}\right) \gamma\left(\tau_{2}^{\prime}\right)+|w| \gamma\left(\tau_{2}^{\prime}\right)\right)=|w| \gamma\left(\tau_{1}^{\prime}\right) \gamma\left(\tau_{2}\right)\left(|w| \gamma\left(\tau_{2}\right)+1\right) .
$$

Since $\gamma$ is bounded by $1 /|w|$ on $B, \gamma\left(\tau_{2}\right)$ is bounded by $1 /|w|^{l}$, and we can conclude about (2.13). In particular, we have

$$
D_{\tau}\left(e^{-i w D_{u} F}-1\right)=O(|w| \gamma(u) \gamma(\tau))
$$

Now define

$$
H=\int_{B}\left|e^{i w D_{u} F}-1\right|^{2} d \lambda^{-}(u) .
$$

By applying the operator $D_{\tau}$ to

$$
\left|e^{i w D_{u} F}-1\right|^{2}=\left(e^{i w D_{u} F}-1\right)\left(e^{-i w D_{u} F}-1\right)
$$

and by using our estimation (2.14) and Lemma 2.7(ii), we obtain

$$
D_{\tau}\left(\left|e^{i w D_{u} F}-1\right|^{2}\right)=O\left(w^{2} \gamma(\tau) \gamma(u)^{2}\right)
$$

So

$$
D_{\tau} H=O\left(w^{2} \gamma(\tau) \int_{B} \gamma(u)^{2} d \lambda^{-}(u)\right)
$$


On the other hand, our assumption (2.6) implies that

$$
H^{-1} \circ \varepsilon_{\tau}^{+}=O\left(\left(w^{2} \int_{B} \gamma(u)^{2} d \lambda^{-}(u)\right)^{-1}\right)
$$

so, from Lemma 2.7(iii),

$$
D_{\tau}(1 / H)=O\left(\gamma(\tau)\left(w^{2} \int_{B} \gamma(u)^{2} d \lambda^{-}(u)\right)^{-1}\right)
$$

Now $Z_{u}$ is the product of the two processes which have been studied in (2.14) and (2.15), so we can conclude from Lemma 2.7(ii).

Proof of Theorem 2.1. The process $\widetilde{Z}\left(\tau_{1}, \tau_{2}, \tau_{2}\right)$ is defined by symmetrization of $(2.10)$, so it is a linear combination of products of processes; in particular,

$$
\left|\widetilde{Z}\left(\tau_{1}, \tau_{2}, \tau_{2}\right)\right| \leq \prod_{u \in \tau_{1}}\left|Z_{u}\right| \prod_{u \in \tau_{2}}\left|Z_{u}\right|^{2}
$$

almost everywhere, so

$$
\widetilde{Z}\left(\tau_{1}, \tau_{2}, \tau_{2}\right)=O\left(\gamma\left(\tau_{1}\right) \gamma\left(\tau_{2}\right)^{2}\left(w \int_{B} \gamma(u)^{2} d \lambda^{-}(u)\right)^{-2 n}\right)
$$

We apply the operator $D_{\tau_{1}}$ and the transformation $\varepsilon_{\tau_{2}}^{+}$to this expression, and we expand $D_{\tau_{1}}$ by iteration of (1.13); we obtain expressions of type $D_{\tau_{1}^{\prime}}\left(Z_{u} \circ \varepsilon_{\tau_{3}}^{-}\right) \circ \varepsilon_{\tau_{2}}^{+}$where $\tau_{1}^{\prime}$ is extracted from $\tau_{1}, u$ and $\tau_{3}$ are extracted from $\left(\tau_{1}, \tau_{2}\right)$; if $\tau_{1}^{\prime}$ and $\tau_{3}$ have a common component, this expression is 0 ; otherwise, it is almost everywhere equal to $D_{\tau_{1}^{\prime}} Z_{u} \circ \varepsilon_{\tau_{2}^{\prime}}^{+}$ where $\tau_{2}^{\prime}$ consists of the components of $\tau_{2}$ which are not in $\tau_{3}$; from Lemmas 2.8 and $2.7(\mathrm{i})$, it is of order

$$
\gamma(u) \gamma\left(\tau_{1}^{\prime}\right)\left(|w| \int_{B} \gamma(v)^{2} d \lambda^{-}(v)\right)^{-1}
$$

This means that applying $D_{\tau_{1}^{\prime}}$ and $\varepsilon_{\tau_{2}^{\prime}}^{+}$multiplies the order of $Z_{u}$ by $\gamma\left(\tau_{1}^{\prime}\right)$. By taking into account all the terms, one can check that applying $D_{\tau_{1}}$ and $\varepsilon_{\tau_{2}}^{+}$to $\widetilde{Z}\left(\tau_{1}, \tau_{2}, \tau_{2}\right)$ multiplies its order by $\gamma\left(\tau_{1}\right)$, so that

$$
D_{\tau_{1}} \widetilde{Z}\left(\tau_{1}, \tau_{2}, \tau_{2}\right) \circ \varepsilon_{\tau_{2}}^{+}=O\left(\gamma\left(\tau_{1}\right)^{2} \gamma\left(\tau_{2}\right)^{2}\left(w \int_{B} \gamma(u)^{2} d \lambda^{-}(u)\right)^{-2 n}\right)
$$

After the integration with respect to $\tau_{1}$ and $\tau_{2}$, we obtain

$$
\begin{aligned}
\int D_{\tau_{1}} \widetilde{Z}\left(\tau_{1}, \tau_{2}, \tau_{2}\right) \circ \varepsilon_{\tau_{2}}^{+} d \lambda^{-}\left(\tau_{1}\right) d \lambda^{-}\left(\tau_{2}\right) & =O\left(w^{-2 n}\left(\int_{B} \gamma(u)^{2} d u\right)^{2 k+l-2 n}\right) \\
& =O\left(w^{-2 n}\left(\int_{B} \gamma(u)^{2} d u\right)^{-n}\right) \\
& =O\left(|w|^{-(2-\alpha / \beta) n}\right)
\end{aligned}
$$

from (2.5). From Lemma 2.6, the left hand side is involved in the variance of $\delta^{(n)}(Z)$, so, by choosing $\zeta$ small enough, the characteristic function $\phi$ of $F$ satisfies

$$
|\phi(w)| \leq \mathbb{E}\left[\left|\delta^{(n)}(Z)\right|^{2}\right]^{1 / 2} \leq C_{n}|w|^{-(2-\alpha / \beta) n / 2},
$$

and we can conclude as in $\S 1$. 


\section{Functionals of Lévy processes}

Suppose that $U=\mathbb{R}_{+} \times \mathbb{R}^{m}$, that $\lambda^{-}$is the product of the Lebesgue measure on $\mathbb{R}_{+}$and a measure $\mu$ on $\mathbb{R}^{m}$ which integrates the function $|x|^{2} \wedge 1$. Then variables on $\Omega$ are functionals of the Lévy process $\left(X_{t} ; t \geq 0\right)$ with Lévy measure $\mu$ defined by $(0.1)$. We would like to find conditions which are sufficient for the existence of a smooth density and which are more tractable than those of Theorem 2.2; these conditions will then be applied to the case of stochastic differential equations. The particular form of $U$ makes possible the use of the differential calculus on $\mathbb{R}^{m}$, and we are going to replace the non-degeneracy condition (2.3) by a condition involving an analogue of the classical Malliavin matrix. A random variable $F$ defined on $\Omega$ can be viewed as a functional of the path $s \mapsto X_{s}$, and for $(t, x) \in U$, the variable $F \circ \varepsilon_{t x}^{+}$is equal to the functional $F$ computed for the transformed path

$$
s \mapsto X_{s}+x 1_{\{s \geq t\}}
$$

Theorem 3.1. Suppose that the Lévy measure $\mu$ satisfies the conditions of Corollary 1.2(b). Let $T>0$ and let $F$ be a $\mathbb{R}^{d}$ valued functional of $\left(X_{t} ; 0 \leq t \leq T\right)$ satisfying

(a) for any $p$ and $k$,

$$
\left\|\operatorname{ess} \sup \left\{\left|D_{\tau} F\right| /\left(\prod_{j=1}^{k}\left|x_{j}\right|\right) ; \tau=\left(\left(t_{1}, x_{1}\right), \ldots,\left(t_{k}, x_{k}\right)\right),\left|x_{j}\right| \leq 1\right\}\right\|_{p}<\infty
$$

(b) there exists a matrix-valued process $\psi_{t}$ such that for $|x| \leq 1, p \geq 1$,

$$
\left\|D_{t x} F-\psi_{t} x\right\|_{p} \leq C_{p}|x|^{r}
$$

for some $r>1$, and

$$
\left\|\left(\operatorname{det} \int_{0}^{T} \psi_{t} \psi_{t}^{\star} d t\right)^{-1}\right\|_{p}<\infty
$$

Then $F$ has a $C_{b}^{\infty}$ density.

Remark. In (a), the essential supremum is relative to the product of measures $d t_{j} d \mu\left(x_{j}\right)$; in particular, we can replace the process $\tau \mapsto D_{\tau} F$ by one of its modifications; this is useful because $F \circ \varepsilon_{\tau}^{+}$is generally not well defined when $X$ has a jump at one of the times $t_{j}$.

In order to prove Theorem 3.1, we have to verify the conditions of Theorem 2.2 ; to this end, we put

$$
\gamma(t, x)=|x| 1_{[0, T]}(t)
$$

and fix $\beta$ in $(\alpha / 2,1)$. We will need the following result which is proved like Lemma 2.7. 
Lemma 3.2. Let $H, H_{1}, H_{2}$ be variables which may depend on some parameters.

(i) If

$$
\left\|\operatorname{esssup}_{\tau \in A(1)^{k}} \frac{\left|D_{\tau} H\right|}{\gamma(\tau)}\right\|_{p} \leq C_{p, k}
$$

for any $k$ and $p$, then

$$
\left\|_{\left(\tau_{1}, \tau_{2}\right) \in A(1)^{k+l}} \frac{\left|D_{\tau_{1}} H\right| \circ \varepsilon_{\tau_{2}}^{+}}{\gamma\left(\tau_{1}\right)}\right\|_{p} \leq C_{p, k, l}
$$

(ii) If

$$
\left\|\operatorname{essips}_{\tau \in A(1)^{k}} \frac{\left|D_{\tau} H_{j}\right|}{\gamma(\tau)}\right\|_{p} \leq C_{p, k}
$$

for $j=1,2$, then

$$
\left\|\operatorname{esssup}_{\tau \in A(1)^{k}} \frac{\left|D_{\tau}\left(H_{1} H_{2}\right)\right|}{\gamma(\tau)}\right\|_{p} \leq C_{p, k}^{\prime}
$$

Now, for $k$ and $v$ fixed, define the events

$$
\begin{aligned}
E_{1}(\rho)= & \left\{\left.\operatorname{essiup}_{\tau \in A(\rho)^{k}}\left|\int_{A(\rho)}\right| D_{u} F \cdot v\right|^{2} \circ \varepsilon_{\tau}^{+} d \lambda^{-}(u)-\int_{A(\rho)}\left|\psi_{t} x \cdot v\right|^{2} d t d \mu(x) \mid\right. \\
& \left.\geq \frac{1}{2} \int_{A(\rho)}\left|\psi_{t} x \cdot v\right|^{2} d t d \mu(x)\right\}
\end{aligned}
$$

and

$$
E_{2}(\rho)=\left\{\operatorname{essip}_{(\tau, u) \in A(\rho)^{k+1}}\left|D_{u} F\right| \circ \varepsilon_{\tau}^{+}>\rho^{\beta}\right\}
$$

and let

$$
E(\rho)=E_{1}(\rho) \cup E_{2}(\rho)
$$

Note that on the complement of $E(\rho)$,

$$
\left(\int_{A(\rho)}\left|D_{u} F \cdot v\right|^{2} 1_{\left\{\left|D_{u} F \cdot v\right| \leq \rho^{\beta}\right\}} d \lambda^{-}(u)\right) \circ \varepsilon_{\tau}^{+} \geq \frac{1}{2} \int_{A(\rho)}\left|\psi_{t} x . v\right|^{2} d t d \mu(x) .
$$

Lemma 3.3. For any $q$,

$$
\mathbb{P}[E(\rho)] \leq C_{k, q} \rho^{q}
$$

Proof. We have to estimate the probabilities of $E_{1}(\rho)$ and $E_{2}(\rho)$ as $\rho \rightarrow 0$. Note that from the assumptions on $V(\rho)$ in Corollary 1.2(b),

$$
\begin{aligned}
\int_{A(\rho)}\left|\psi_{t} x . v\right|^{2} d t d \mu(x) & =v^{\star} \int_{0}^{T} \psi_{t}\left(\int_{\{|x| \leq \rho\}} x x^{\star} d \mu(x)\right) \psi_{t}^{\star} d t v \\
& \geq c \int_{\{|x| \leq \rho\}}|x|^{2} d \mu(x) \Lambda\left(\int_{0}^{T} \psi_{t} \psi_{t}^{\star} d t\right)
\end{aligned}
$$


where $\Lambda(S)$ denotes the smallest eigenvalue of $S$, so from our assumption (3.3),

$$
\left(\int_{A(\rho)}\left|\psi_{t} x \cdot v\right|^{2} d t d \mu(x)\right)^{-1}=O\left(\left(\int_{\{|x| \leq \rho\}}|x|^{2} d \mu(x)\right)^{-1}\right)
$$

in the spaces $L^{p}$; on the other hand,

$$
\begin{aligned}
& \left|\int_{A(\rho)}\right| D_{u} F .\left.v\right|^{2} \circ \varepsilon_{\tau}^{+} d \lambda^{-}(u)-\int_{A(\rho)}\left|\psi_{t} x . v\right|^{2} d t d \mu(x) \mid \\
& \leq \int_{A(\rho)}\left|\bar{D}_{\tau}\left(\left|D_{u} F . v\right|^{2}\right)\right| d \lambda^{-}(u)+\int_{A(\rho)}\left(2\left|\psi_{t} x\right|+\left|D_{t x} F-\psi_{t} x\right|\right)\left|D_{t x} F-\psi_{t} x\right| d t d \mu(x)
\end{aligned}
$$

with the notation

$$
\bar{D}_{\tau} H=H \circ \varepsilon_{\tau}^{+}-H=\sum_{j=1}^{k} D_{u_{j}} H \circ \varepsilon_{u_{1}}^{+} \circ \ldots \circ \varepsilon_{u_{j-1}}^{+} .
$$

One deduces from (3.1) and Lemma 3.2 that

$$
\left\|_{\left(u_{1}, \ldots, u_{k}\right) \in A(1)^{k}}\left|D_{u_{j}}\left(\left|D_{u} F . v\right|^{2}\right) \circ \varepsilon_{u_{1}}^{+} \circ \ldots \circ \varepsilon_{u_{j-1}}^{+}\right| /\left(\gamma\left(u_{j}\right) \gamma(u)^{2}\right)\right\|_{p} \leq C_{p, k}
$$

so

$$
\operatorname{ess~sup}_{\tau \in A(\rho)^{k}}\left|\bar{D}_{\tau}\left(\left|D_{u} F . v\right|^{2}\right)\right|=O\left(\rho \gamma(u)^{2}\right) .
$$

From (3.2), (3.7) and (3.8), one obtains

$$
\begin{aligned}
& \left.\operatorname{esssup}_{\tau \in A(\rho)^{k}}\left|\int_{A(\rho)}\right| D_{u} F \cdot v\right|^{2} \circ \varepsilon_{\tau}^{+} d \lambda^{-}(u)-\int_{A(\rho)}\left|\psi_{t} x . v\right|^{2} d t d \mu(x) \mid \\
& =O\left(\rho \int_{A(\rho)} \gamma(u)^{2} d \lambda^{-}(u)+\int_{A(\rho)} \gamma(u)^{r+1} d \lambda^{-}(u)\right) \\
& =O\left(\rho^{(r-1) \wedge 1} \int_{\{|x| \leq \rho\}}|x|^{2} d \mu(x)\right)
\end{aligned}
$$

Thus the product of the left-hand sides of (3.6) and (3.9) is of order $\rho^{(r-1) \wedge 1}$ in any $L^{p}$, so, since $E_{1}(\rho)$ is the set of $\omega \in \Omega$ such that this product is greater than $1 / 2$, its probability is of order $\rho^{q}$ for any $q>0$. In order to estimate $E_{2}(\rho)$, one deduces from (3.1) and Lemma $3.2(\mathrm{i})$ that

$$
\left\|_{(\tau, u) \in A(1)^{k+1}} \frac{\left|D_{u} F\right| \circ \varepsilon_{\tau}^{+}}{\gamma(u)}\right\|_{p} \leq C_{p, k}
$$

so

$$
\operatorname{ess~sup}_{(\tau, u) \in A(\rho)^{k+1}} \frac{\left|D_{u} F\right| \circ \varepsilon_{\tau}^{+}}{\rho^{\beta}}=O\left(\rho^{1-\beta}\right)
$$


and therefore, the probability of $E_{2}(\rho)$ is also of order $\rho^{q}$ for any $q>0$.

Proof of Theorem 3.1. Recall that $\gamma(t, x)$ is $|x|$ for $t \in[0, T]$, so condition (2.1) is evident from the assumption (1.4); thus we have to verify that each variable $F . v, v \in S^{d-1}$, satisfies (2.2) and (2.3). But (2.2) follows immediately from (3.1), so let us prove (2.3) for $p$ and $k$ fixed. Define

$$
R=R(\rho, \omega)=\sup \left\{\rho^{\prime} \leq \rho ; \quad \omega \notin E\left(\rho^{\prime}\right)\right\}
$$

From Lemma 3.3,

$$
\forall \rho^{\prime} \leq \rho \quad \mathbb{P}\left[R<\rho^{\prime}\right] \leq \mathbb{P}\left[E\left(\rho^{\prime}\right)\right] \leq C_{q} \rho^{\prime q} .
$$

From the definition of $R$, there exists a variable $R^{\prime}=R^{\prime}(\rho, \omega)$ taking the value $\rho$ on the complement of $E(\rho)$, and such that

$$
R(\rho, \omega) / 2 \leq R^{\prime}(\rho, \omega) \leq R(\rho, \omega) \quad \text { and } \quad \omega \notin E\left(R^{\prime}(\rho, \omega)\right) .
$$

One can check that $R^{\prime}$ also satisfies an estimate of type (3.10), so the moments of $1 / R^{\prime}$ are finite, and moreover

$$
\mathbb{E}\left[R^{\prime-n} 1_{\left\{R^{\prime}<\rho\right\}}\right] \leq C_{n, q} \rho^{q}
$$

for any $n$ and $q$. Now if we recall that $\Lambda(S)$ denotes the smallest eigenvalue of $S$,

$$
\begin{aligned}
& \left(\int_{A(\rho)}\left|D_{u} F \cdot v\right|^{2} 1_{\left\{\left|D_{u} F \cdot v\right| \leq \rho^{\beta}\right\}} d \lambda^{-}(u)\right)^{-1} \circ \varepsilon_{\tau}^{+} \\
& \leq\left(\int_{A\left(R^{\prime}\right)}\left|D_{u} F \cdot v\right|^{2} 1_{\left\{\left|D_{u} F \cdot v\right| \leq \rho^{\beta}\right\}} d \lambda^{-}(u)\right)^{-1} \circ \varepsilon_{\tau}^{+} \\
& \leq 2\left(\int_{A\left(R^{\prime}\right)}\left|\psi_{t} x \cdot v\right|^{2} d t d \mu(x)\right)^{-1} \\
& \leq C \Lambda\left(\int_{0}^{T} \psi_{t} \psi_{t}^{\star} d t\right)^{-1}\left(\int_{\left\{|x| \leq R^{\prime}\right\}}|x|^{2} d \mu(x)\right)^{-1} \\
& \leq C \Lambda\left(\int_{0}^{T} \psi_{t} \psi_{t}^{\star} d t\right)^{-1}\left[\left(\int_{\{|x| \leq \rho\}}|x|^{2} d \mu(x)\right)^{-1}+C R^{\prime-\alpha} 1_{\left\{R^{\prime}<\rho\right\}}\right] .
\end{aligned}
$$

In the first inequality, we have used the property $R^{\prime} \leq \rho$; in the second one, we have used (3.4); in the third one, we have used (3.5); in the last one, we have considered separately the cases $R^{\prime}=\rho$ and $R^{\prime}<\rho$, and in the latter case, we have applied

$$
\int_{\left\{|x| \leq R^{\prime}\right\}}|x|^{2} d \mu(x) \geq c R^{\prime \alpha} .
$$

Thus the estimation (2.3) which has to be proved for F.v can be reduced to the estimation of

$$
\left(\int_{\{|x| \leq \rho\}}|x|^{2} d \mu(x)\right)^{-1}+C\left\|R^{\prime-\alpha} 1_{\left\{R^{\prime}<\rho\right\}}\right\|_{p}
$$

as $\rho \rightarrow 0$. The first term (which tends to infinity) is exactly what we want, and the second one is negligible from (3.11). 
Remark. Suppose that $X_{t}$ has not approximately the same number of jumps in all the directions, so that eigenvalues of $V(\rho)$ are not in a bounded ratio. Then one can still obtain a similar result, provided that the nondegeneracy condition (3.3) holds in the directions where there are many jumps. More precisely, if

$$
\int_{\{|x| \leq \rho\}} x x^{\star} d \mu(x) \geq S \int_{\{|x| \leq \rho\}}|x|^{2} d \mu(x)
$$

for some symmetric positive semidefinite matrix $S$, then conclusion holds if

$$
\left\|\left(\operatorname{det} \int_{0}^{T} \psi_{t} S \psi_{t}^{\star} d t\right)^{-1}\right\|_{p}<\infty
$$

One can also apply a partial calculus; if $\mu=\mu_{1}+\mu_{2}$, then $\lambda^{+}$can be decomposed into the sum of two independent Poisson measures; if one applies our calculus only with respect to the first one, then only $\mu_{1}$ has to satisfy our assumption.

\section{The case of stochastic differential equations}

Consider again the Lévy process $X_{t}$ of (0.1), and let us study the special case $F=Y_{T}$ of a variable defined by a $X$-driven stochastic differential equation; we want to see how conditions of Theorem 3.1 can be verified in this framework. Let $a(y, x)$ and $b(y)$ be $\mathbb{R}^{d}$ valued functions defined respectively on $\mathbb{R}^{d} \times \mathbb{R}^{m}$ and $\mathbb{R}^{d}$; suppose that

$$
a(y, x)=\bar{a}(y) x+\widetilde{a}(y, x)
$$

where $\widetilde{a}(y, x)=o(|x|)$ as $x \rightarrow 0$. We will say that the process $Y_{t}$ is a solution of

$$
d Y_{t}=b\left(Y_{t}\right) d t+a\left(Y_{t-}, d X_{t}\right), \quad Y_{0}=y_{0},
$$

if

$$
Y_{t}=y_{0}+\int_{0}^{t} b\left(Y_{s}\right) d s+\int_{0}^{t} \bar{a}\left(Y_{s-}\right) d X_{s}+\sum_{s \leq t} \widetilde{a}\left(Y_{s-}, \Delta X_{s}\right)
$$

where

$$
\sum_{s \leq t}\left|\widetilde{a}\left(Y_{s-}, \Delta X_{s}\right)\right|<\infty
$$

If $g(y, x)$ is smooth with respect to $y$ and if $k \in \mathbb{N}^{d}$, the function $g$ differentiated $k_{j}$ times with respect to each $y_{j}$ is denoted by $g^{(k)}$; the Jacobian matrix with respect to $y$ is denoted by $g^{\prime}$. Henceforth, the regularity and boundedness assumptions for $a$ and $b$ are the following ones.

Assumption (A). The functions $\bar{a}$ and $b$ are $C^{\infty}$ with bounded derivatives, the function $\widetilde{a}(y, x)$ is infinitely differentiable with respect to $y$, and

$$
|\widetilde{a}(y, x)| \leq C(1+|y|)|x|^{r}, \quad\left|\widetilde{a}^{(k)}(y, x)\right| \leq C_{k}|x|^{r}
$$


for $k \in \mathbb{N}^{d} \backslash\{0\},|x| \leq 1$, and some $r>1$ such that

$$
\int\left(|x|^{r} \wedge 1\right) d \mu(x)<\infty
$$

Moreover

$$
\int|a(y, x)|^{p} d \mu(x) \leq C(1+|y|)^{p}, \quad \sup _{y} \int\left|a^{(k)}(y, x)\right|^{p} d \mu(x)<\infty
$$

for $k \in \mathbb{N}^{d} \backslash\{0\}, p \geq 2$.

Under Assumption (A), the equation (4.1) has a unique solution $Y_{t}$. When $\widetilde{a}=0$, this is a standard result since the coefficients $\bar{a}$ and $b$ are globally Lipschitz. The general case can be dealt with by writing (4.1) as $d Y_{t}=d \widetilde{X}_{t}\left(Y_{t-}\right)$ in the sense of [4], where

$$
\widetilde{X}_{t}(y)=b(y) t+\int_{0}^{t} a\left(y, d X_{s}\right)=b(y) t+\bar{a}(y) X_{t}+\sum_{s \leq t} \widetilde{a}\left(y, \Delta X_{s}\right)
$$

is a Lévy process with values in the space of smooth maps from $\mathbb{R}^{d}$ into itself (the convergence and smoothness of the sum follow from (4.2) and (4.3)).

Moreover, it is proved in [4] that for $(t, y)$ fixed, the solution at time $s \geq t$ with initial value $y$ at time $t$ is given by a smooth stochastic semiflow $\phi_{t s}(y)$, and the derivatives of $\phi_{t s}$ are obtained by deriving formally the equation (in [1], a weaker differentiability is studied; when $\widetilde{a}=0$, one can also see [9]). In particular, the derivative $Z_{s}^{t}=\phi_{t s}^{\prime}\left(Y_{t}\right)$ is the unique matrix-valued solution of

$$
d Z_{s}^{t}=b^{\prime}\left(Y_{s}\right) Z_{s}^{t} d s+a^{\prime}\left(Y_{s-}, d X_{s}\right) Z_{s-}^{t}, \quad Z_{t}^{t}=I
$$

for $s \geq t$. In contrast with the continuous case, one must notice that the semiflow $\phi_{t s}$ is invertible only when $\mu$-almost all the maps

$$
y \mapsto y+a(y, x)
$$

are invertible (see for instance [5] in the case $\widetilde{a}=0$ ).

We now verify that in the case $F=Y_{T}$, the Malliavin matrix involved in Theorem 3.1 can be expressed as in the classical continuous case; the analogue of the diffusion coefficient is $\bar{a} \bar{a}^{\star}$.

Theorem 4.1. Assume that $a$ and $b$ satisfy (A), that the Lévy measure $\mu$ of $X$ satisfies the conditions of Corollary 1.2(b), and that the nondegeneracy condition (3.3) holds with

$$
\psi_{t}=Z_{T}^{t} \bar{a}\left(Y_{t}\right)
$$


for some $T>0$. Then $Y_{T}$ has a $C_{b}^{\infty}$ density.

We need some preliminary results. We will use the Sobolev inequality

$$
\sup |H(y)| \leq C \sum_{|k|=d+1} \int\left|H^{(k)}(y)\right| d y
$$

which is valid for smooth functions $H$ with compact support in $\mathbb{R}^{d}$, and which is easily proved by estimating the Fourier transform of $H$; by localizing, one deduces that

$$
\sup _{|y| \leq \rho}|H(y)| \leq C \sum_{|k| \leq d+1} \int_{\{|y| \leq \rho+1\}}\left|H^{(k)}(y)\right| d y
$$

for a $C$ which does not depend on $\rho$.

Lemma 4.2. Let $H_{1}\left(\omega, y_{1}, z\right)$ and $H_{2}\left(\omega, y_{1}, y, z\right), y_{1} \in \mathbb{R}^{d}, y \in \mathbb{R}^{d}, z \in E$ (a parameter space), be random functions such that

$$
\begin{gathered}
\left\|\sup _{z}\left|H_{1}\left(y_{1}, z\right)\right|\right\|_{p} \leq Q_{p}\left(y_{1}\right), \\
\left\|\sup _{z}\left|H_{2}^{(k)}\left(y_{1}, y, z\right)\right|\right\|_{p} \leq Q_{k p}(y),
\end{gathered}
$$

for $p \geq 1, k \in \mathbb{N}^{d}$, some functions $Q_{p}, Q_{k p}$ with at most polynomial growth, and where $H_{2}^{(k)}$ are the derivatives with respect to $y$. Then the function

$$
H:\left(y_{1}, z\right) \mapsto H_{2}\left(y_{1}, H_{1}\left(y_{1}, z\right), z\right)
$$

satisfies an estimate similar to the one for $H_{1}$ : for any $p$, there exists a function $\bar{Q}_{p}$ with at most polynomial growth such that

$$
\left\|\sup _{z}\left|H\left(y_{1}, z\right)\right|\right\|_{p} \leq \bar{Q}_{p}\left(y_{1}\right)
$$

Proof. By applying the Sobolev inequality (4.7) to $y \mapsto H_{2}\left(y_{1}, y, z\right)$,

$$
\begin{aligned}
\left|H\left(y_{1}, z\right)\right| & \leq \sup \left\{\left|H_{2}\left(y_{1}, y, z\right)\right| ;|y| \leq \sup _{z}\left|H_{1}\left(y_{1}, z\right)\right|\right\} \\
& \leq C \sum_{|k| \leq d+1} \int_{\left\{|y| \leq \sup _{z}\left|H_{1}\left(y_{1}, z\right)\right|+1\right\}}\left|H_{2}^{(k)}\left(y_{1}, y, z\right)\right| d y .
\end{aligned}
$$

Thus

$$
\begin{aligned}
& \left\|\sup _{z}\left|H\left(y_{1}, z\right)\right|\right\|_{p} \\
& \leq C \sum_{|k| \leq d+1} \int\left\|\sup _{z}\left|H_{2}^{(k)}\left(y_{1}, y, z\right)\right|\right\|_{2 p} \mathbb{P}\left[\sup _{z}\left|H_{1}\left(y_{1}, z\right)\right|+2 \geq|y|+1\right]^{1 /(2 p)} d y \\
& \leq C\left(Q_{q}\left(\left|y_{1}\right|\right)+2\right)^{q / 2 p} \sum_{|k| \leq d+1} \int(|y|+1)^{-q / 2 p} Q_{k, 2 p}(y) d y
\end{aligned}
$$

where the probability was estimated from the Bienaymé-Chebyshev inequality. By choosing $q$ large enough, the integrals are finite, so we can conclude. 
Lemma 4.3. One has

$$
\left\|\sup _{0 \leq s \leq t \leq T}\left|\phi_{s t}^{(k)}(y)\right|\right\|_{p} \leq Q_{k p}(y)
$$

for some functions $Q_{k p}$ with at most polynomial growth, and where the supremum is relative to the couples $(s, t)$.

Proof. If we only take the supremum with respect to $t$ with $s$ fixed, this is a standard estimate on the solution of (4.1), and $Q_{k p}$ is affine if $k=0$ and is constant otherwise (see [4]; estimates of the derivatives require the graded equations of [1]). More generally, for any stopping time $\sigma$, the process $\phi_{\sigma t}(y)$ is the solution of (4.1) with initial value $y$ at time $\sigma$, and

$$
\left\|\sup _{\sigma \leq t \leq T}\left|\phi_{\sigma t}(y)\right|\right\|_{p} \leq C_{p}(1+|y|), \quad\left\|\sup _{\sigma \leq t \leq T}\left|\phi_{\sigma t}^{(k)}(y)\right|\right\|_{p} \leq C_{p}
$$

for $k \neq 0$ and where $C_{p}$ does not depend on $\sigma$. The delicate point in the lemma is that we have to take also the supremum with respect to $s$; moreover, the flow is not necessarily invertible, so we cannot write $\phi_{s t}=\phi_{t} \phi_{s}^{-1}$. To make it invertible, we have to remove the big jumps. Let $\rho_{0}>0$ be such that

$$
|x| \leq \rho_{0} \Longrightarrow \sup _{y}\left|a^{\prime}(y, x)\right| \leq 1 / 2
$$

When $|x| \leq \rho_{0}$, the map $y \mapsto y+a(y, x)$ is a diffeomorphism, so $\phi_{s t}$ is a diffeomorphism as soon as $X_{t}$ has not a jump bigger than $\rho_{0}$ on $[s, t]$ (see [5], [4]). Now consider the sequence of stopping times $\sigma_{j}$ where $\sigma_{0}=0$ and $\sigma_{j+1}$ is the infimum of times $s>\sigma_{j}$ such that

$$
\left|\Delta X_{s}\right| \geq \rho_{0} \quad \text { or } \quad \sup _{\left|y-y_{1}\right| \leq \rho_{1}}\left|\phi_{\sigma_{j} s}(y)-y\right| \geq \rho_{1} / 2 \quad \text { or } \quad s \geq \sigma_{j}+T
$$

for fixed $\rho_{1}>0$ and $y_{1}$. On the event $\left\{\sigma_{j} \leq s<\sigma_{j+1}\right\}$, the map $\phi_{\sigma_{j} s}$ is invertible and we can write

$$
\phi_{s t}=\phi_{\sigma_{j} t} \circ \phi_{\sigma_{j} s}^{-1}
$$

We want to estimate $\phi_{s t}^{(k)}\left(y_{1}\right)$ uniformly in $s \in\left[\sigma_{j}, \sigma_{j+1}\right)$ and $t \in[s, T]$. We expand this derivative as a sum of products of type

$$
\phi_{\sigma_{j} t}^{(k)} \circ \phi_{\sigma_{j} s}^{-1}\left(y_{1}\right), \quad \phi_{\sigma_{j} s}^{(k)} \circ \phi_{\sigma_{j} s}^{-1}\left(y_{1}\right), \quad\left(\phi_{\sigma_{j} s}^{\prime}\right)^{-1} \circ \phi_{\sigma_{j} s}^{-1}\left(y_{1}\right) .
$$

From Lemma 4.2, we are reduced to estimate the $L^{p}$ norms of

$$
\sup _{\sigma_{j} \leq t \leq T}\left|\phi_{\sigma_{j} t}^{(k)}(y)\right|, \quad \sup _{\sigma_{j} \leq s<\sigma_{j+1}}\left|\left(\phi_{\sigma_{j} s}^{\prime}\right)^{-1}(y)\right|, \quad \sup _{\sigma_{j} \leq s<\sigma_{j+1}}\left|\phi_{\sigma_{j} s}^{-1}\left(y_{1}\right)\right|
$$

by some functions of $y$ or $y_{1}$ with at most polynomial growth, and the estimates for the first and second terms should not depend on $y_{1}$ (note that $\sigma_{j}$ depends on $y_{1}$ ). Firstly 
$\phi_{\sigma_{j} t}^{(k)}(y)$ is estimated in (4.8). Secondly, $\phi_{\sigma_{j} s}^{\prime}(y)$ is solution of (4.5) with $Y_{s}$ replaced by $\phi_{\sigma_{j} s}(y)$, so $\left(\phi_{\sigma_{j} s}^{\prime}(y)\right)^{-1}$ is solution of

$$
d \bar{Z}_{s}=-\bar{Z}_{s}\left(b^{\prime} \circ \phi_{\sigma_{j} s}\right)(y) d s+\bar{Z}_{s}\left(\left(I+a^{\prime}\left(\phi_{\sigma_{j} s-}(y), d X_{s}\right)\right)^{-1}-I\right)
$$

Since the coefficients of this equation are bounded, one can deduce that

$$
\left\|\sup _{\sigma_{j} \leq s<\sigma_{j+1}}\left|\left(\phi_{\sigma_{j} s}^{\prime}(y)\right)^{-1}\right|\right\|_{p} \leq C_{p}
$$

Thirdly, we have to study $\phi_{\sigma_{j} s}^{-1}\left(y_{1}\right)$ on $\left\{\sigma_{j} \leq s<\sigma_{j+1}\right\}$; but from the definition of $\sigma_{j+1}$, we have that

$$
\left|y-y_{1}\right| \leq \rho_{1} \Longrightarrow\left|\phi_{\sigma_{j} s}(y)-y\right|<\rho_{1} / 2,
$$

so the image by $\phi_{\sigma_{j} s}$ of the sphere of center $y_{1}$ and radius $\rho_{1}$ is included in the complement of the closed ball of center $y_{1}$ and radius $\rho_{1} / 2$ and this ball contains $\phi_{\sigma_{j} s}\left(y_{1}\right)$; since $\phi_{\sigma_{j} s}$ is a diffeomorphism, the image of the ball of center $y_{1}$ and radius $\rho_{1}$ contains the ball of center $y_{1}$ and radius $\rho_{1} / 2$, so

$$
\left|\phi_{\sigma_{j} s}^{-1}\left(y_{1}\right)-y_{1}\right| \leq \rho_{1}
$$

and therefore

$$
\left\|\sup _{\sigma_{j} \leq s<\sigma_{j+1}}\left|\phi_{\sigma_{j} s}^{-1}\left(y_{1}\right)\right|\right\|_{p} \leq y_{1}+\rho_{1} .
$$

As it was explained above, from (4.8), (4.12) and (4.13), we can apply Lemma 4.2 and deduce that

$$
\left\|\sup _{0 \leq s \leq t \leq T}\left|\phi_{s t}^{(k)}\left(y_{1}\right)\right| 1_{\left\{\sigma_{j} \leq s<\sigma_{j+1}\right\}}\right\|_{p} \leq Q_{k, p}\left(y_{1}\right)
$$

for $Q_{k, p}$ with at most polynomial growth. Now

$$
\begin{aligned}
\left\|\sup _{0 \leq s \leq t \leq T}\left|\phi_{s t}^{(k)}\left(y_{1}\right)\right|\right\|_{p} & \leq \sum_{j}\left\|\sup _{0 \leq s \leq t \leq T}\left|\phi_{s t}^{(k)}\left(y_{1}\right)\right| 1_{\left\{\sigma_{j} \leq s<\sigma_{j+1}\right\}} 1_{\left\{\sigma_{j} \leq T\right\}}\right\|_{p} \\
& \leq Q_{k, 2 p}\left(y_{1}\right) \sum_{j} \mathbb{P}\left[\sigma_{j} \leq T\right]^{1 /(2 p)}
\end{aligned}
$$

so we still have to estimate the series. From the definition of $\left(\sigma_{j}\right)$, it can be seen that the variables $\left(\sigma_{j+1}-\sigma_{j}\right)$ are independent, identically distributed, and that

$$
\mathbb{P}\left[\sigma_{1} \leq t\right] \leq \mathbb{P}\left[\sup _{s \leq t}\left|\Delta X_{s}\right| \geq \rho_{0}\right]+\frac{2}{\rho_{1}} \mathbb{E} \sup _{s \leq t} \sup _{\left|y-y_{1}\right| \leq \rho_{1}}\left|\phi_{s}(y)-y\right|
$$

for $t<T$ and with $\phi_{s}=\phi_{0 s}$. Since $X_{t}$ is a Lévy process,

$$
\mathbb{P}\left[\sup _{s \leq t}\left|\Delta X_{s}\right| \geq \rho_{0}\right] \leq C t
$$


The supremum with respect to $y$ is estimated from the Sobolev inequality (4.7), so that

$$
\begin{aligned}
\sup _{\left|y-y_{1}\right| \leq \rho_{1}}\left|\phi_{s}(y)-y\right| \leq & C \int_{\left\{\left|y-y_{1}\right| \leq \rho_{1}+1\right\}}\left|\phi_{s}(y)-y\right| d y \\
& +C \sum_{1 \leq|k| \leq d+1} \int_{\left\{\left|y-y_{1}\right| \leq \rho_{1}+1\right\}}\left|\phi_{s}^{(k)}(y)\right| d y .
\end{aligned}
$$

We deduce from the $L^{2}$ estimates of [4] that

$$
\mathbb{E} \sup _{s \leq t}\left|\phi_{s}(y)-y\right| \leq C(1+|y|) \sqrt{t}, \quad \mathbb{E} \sup _{s \leq t}\left|\phi_{s}^{(k)}(y)\right| \leq C_{k} \sqrt{t}
$$

for $k \neq 0$, so

$$
\mathbb{P}\left[\sigma_{1} \leq t\right] \leq \sqrt{t} Q\left(y_{1}\right) \leq 1 / 2
$$

if $t<T \wedge 1 /\left(4 Q\left(y_{1}\right)^{2}\right)$, with $Q$ having at most polynomial growth. Let $J=J\left(y_{1}\right)$ be the first integer greater than $4 T Q\left(y_{1}\right)^{2}$; since $\sigma_{j}$ is the sum of $j$ independent variables distributed like $\sigma_{1}$, we have

$$
\mathbb{P}\left[\sigma_{j} \leq T\right] \leq J \mathbb{P}\left[\sigma_{[j / J]} \leq T / J\right] \leq J \mathbb{P}\left[\sigma_{1} \leq T / J\right]^{[j / J]} \leq(2 J) /\left(2^{j / J}\right)
$$

where $[j / J]$ is the integer value of $j / J$. We deduce that

$$
\sum_{j} \mathbb{P}\left[\sigma_{j} \leq T\right]^{1 / 2 p} \leq C_{p}\left(4 T Q\left(y_{1}\right)^{2}+1\right)^{1+1 / 2 p} .
$$

The result now follows from (4.14) and (4.15).

Proof of Theorem 4.1. Consider the function

$$
\xi(\rho, x, y)=y+\rho|x|^{-1} a(y, x), \quad \rho \geq 0, \quad|x| \leq 1,
$$

and for $0 \leq t_{1}<\ldots<t_{k} \leq T$, the random map

$$
\Xi\left(y_{0}, \rho_{1}, t_{1}, x_{1}, \ldots, \rho_{k}, t_{k}, x_{k}\right)=\phi_{t_{k} T} \circ \xi\left(\rho_{k}, x_{k}, .\right) \circ \phi_{t_{k-1} t_{k}} \circ \ldots \circ \xi\left(\rho_{1}, x_{1}, .\right) \circ \phi_{0 t_{1}}\left(y_{0}\right) .
$$

Then for $\tau=\left(t_{1}, x_{1}, \ldots, t_{k}, x_{k}\right)$, one has

$$
Y_{T} \circ \varepsilon_{\tau}^{+}=\Xi\left(y_{0},\left|x_{1}\right|, t_{1}, x_{1}, \ldots,\left|x_{k}\right|, t_{k}, x_{k}\right)
$$

and

$$
D_{\tau} F=\int_{0}^{\left|x_{1}\right|} d \rho_{1} \ldots \int_{0}^{\left|x_{k}\right|} d \rho_{k} \frac{\partial^{k} \Xi}{\partial \rho_{1} \ldots \partial \rho_{k}}\left(y_{0}, \rho_{1}, t_{1}, x_{1}, \ldots, \rho_{k}, t_{k}, x_{k}\right),
$$

so in order to estimate the left-hand side of (3.1), we can use

$$
\begin{aligned}
\underset{\tau}{\operatorname{ess} \sup } \frac{\left|D_{\tau} F\right|}{\prod\left|x_{j}\right|} \leq \sup \left\{\left|\frac{\partial^{k} \Xi}{\partial \rho_{1} \ldots \partial \rho_{k}}\left(y_{0}, \rho_{1}, t_{1}, x_{1}, \ldots, \rho_{k}, t_{k}, x_{k}\right)\right| ;\right. \\
\left.0 \leq \rho_{j} \leq 1,0 \leq t_{1}<\ldots<t_{k} \leq T,\left|x_{j}\right| \leq 1\right\} .
\end{aligned}
$$


Now, by expanding the derivative, since the derivatives of $\xi$ with respect to $\rho$ and $y$ are bounded, we are reduced to estimate the moments of variables of type

$$
\phi_{t_{j} t_{j+1}}^{\left(k^{\prime}\right)} \circ \xi\left(\rho_{j}, x_{j}, .\right) \circ \phi_{t_{j-1} t_{j}} \circ \ldots \circ \xi\left(\rho_{1}, x_{1}, .\right) \circ \phi_{0 t_{1}}\left(y_{0}\right)
$$

We apply Lemma 4.2 to this composed function, and $\phi_{t_{j} t_{j+1}}^{\left(k^{\prime}\right)}(y), \phi_{t_{l} t_{l+1}}(y)$ are estimated from Lemma 4.3, so we obtain the condition (a) of Theorem 3.1. For (b), it is clear that

$$
Y_{T} \circ \varepsilon_{t x}^{+}=\phi_{t T}\left(Y_{t}+a\left(Y_{t}, x\right)\right)
$$

is differentiable with respect to $x$ at $x=0$, and if $\psi_{t}$ is the Jacobian matrix, it is given by (4.6). Moreover

$$
D_{t x} Y_{T}=\Xi\left(y_{0},|x|, t, x\right)-\Xi\left(y_{0}, 0, t, x\right)
$$

and

$$
\psi_{t} x=\sum_{j=1}^{d} \frac{\partial \phi_{t T}}{\partial y_{j}}\left(Y_{t}\right) \bar{a}_{j}\left(Y_{t}\right) x=|x| \frac{\partial \Xi}{\partial \rho}\left(y_{0}, 0, t, x\right)-\sum_{j=1}^{d} \frac{\partial \phi_{t T}}{\partial y_{j}}\left(Y_{t}\right) \widetilde{a}_{j}\left(Y_{t}, x\right)
$$

So

$$
\begin{aligned}
D_{t x} Y_{T}-\psi_{t} x= & \sum_{j, k} \int_{0}^{|x|} d \bar{\rho} \int_{0}^{\bar{\rho}} d \rho \frac{\partial^{2} \phi_{t T}}{\partial y_{j} \partial y_{k}}\left(Y_{t}+\rho \frac{a\left(Y_{t}, x\right)}{|x|}\right) \frac{a_{j}\left(Y_{t}, x\right) a_{k}\left(Y_{t}, x\right)}{|x|^{2}} \\
& +\sum_{j} \frac{\partial \phi_{t T}}{\partial y_{j}}\left(Y_{t}\right) \widetilde{a}_{j}\left(Y_{t}, x\right) .
\end{aligned}
$$

The moments of the first and second derivatives are proved to be bounded from (4.8), the variables $a_{j}\left(Y_{t}, x\right)$ and $\widetilde{a}_{j}\left(Y_{t}, x\right)$ are respectively of order $|x|$ and $|x|^{r}$, so this expression is of order $|x|^{r \wedge 2}$.

In Theorem 4.1, the only condition which does not rely explicitely on the coefficients of the equation is the condition (3.3) concerning the Malliavin matrix; we now want to find sufficient conditions for it; this condition can actually be decomposed into conditions on $Z_{T}^{t}$ and on $\bar{a}$; the condition on $Z_{T}^{t}$ is linked with the local invertibility of the flow (note that such a condition does not appear for continuous diffusions); for $\bar{a}$, we verify that the uniform ellipticity of $\bar{a} \bar{a}^{\star}$ is sufficient, but we also give another example, namely the Lévy stochastic area; this example can probably be generalized to a more general condition of Hörmander's type as in [7], but we will not deal with this problem in this work.

Corollary 4.4. Suppose that $\mu$ satisfies the conditions of Corollary 1.2(b), that $a$ and $b$ satisfy (A), that $\bar{a} \bar{a}^{\star}$ is uniformly elliptic, and that

$$
\operatorname{det}\left(I+a^{\prime}(y, x)\right) \geq c /\left(1+|y|^{q}\right)
$$

for some $q \geq 0$, any $y$ and $\mu$ almost any $x$. Then $Y_{T}$ has a $C_{b}^{\infty}$ density for any $T>0$.

Proof. One can check from the assumption (4.16) that the derivative $Z_{s}^{t}$ of the flow $\phi_{t s}$ is invertible; moreover, if $\sigma_{0}=T$ and

$$
\sigma_{j}=\sup \left\{t<\sigma_{j-1} ;\left|\Delta X_{t}\right| \geq \rho_{0}\right\}
$$


for $\rho_{0}$ defined in (4.9) and with the convention $\sup \emptyset=0$, then

$$
Z_{T}^{t}=Z_{T}^{\sigma_{1}}\left(I+a^{\prime}\left(Y_{\sigma_{1}-}, \Delta X_{\sigma_{1}}\right)\right) Z_{\sigma_{1}-}^{\sigma_{2}} \ldots Z_{\sigma_{j-2}-}^{\sigma_{j-1}}\left(I+a^{\prime}\left(Y_{\sigma_{j-1}-}, \Delta Z_{\sigma_{j-1}}\right)\right) Z_{\sigma_{j-1}-}^{t}
$$

on $\left\{\sigma_{j} \leq t<\sigma_{j-1}\right\}$. Conditionally on $\left(\sigma_{k}, k \in \mathbb{N}\right)$, one can write the equation satisfied by $\left(Z_{t}^{\sigma_{j}}\right)$ on $\left\{\sigma_{j} \leq t<\sigma_{j-1}\right\}$ as an equation similar to (4.5), but with the jumps of $X$ greater than $\rho_{0}$ removed; the solution of this equation is invertible and its inverse satisfies (4.11) with the big jumps of $X$ removed. The times $\sigma_{k}$ are not stopping times, but the sequence $\left(\sigma_{k}\right)$ and the process $X$ with its big jumps removed are independent, so one can deduce as in (4.12) that

$$
\mathbb{E}\left[\sup _{\sigma_{j} \leq t<\sigma_{j-1}}\left|\left(Z_{t}^{\sigma_{j}}\right)^{-1}\right|^{p} \mid\left(\sigma_{k} ; k \in \mathbb{N}\right)\right] \leq C_{p}
$$

From this estimate and (4.16), by writing the inverse $U_{t}$ of $Z_{T}^{t}$ from (4.17), we obtain

$$
\left\|\sup _{t>\sigma_{k}}\left|U_{t}\right|\right\|_{p} \leq C_{k, p}\left\|\sup _{s}\left(1+\left|Y_{s}\right|^{q}\right)^{k-1}\right\|_{p^{\prime}}<\infty
$$

On the other hand

$$
\left(\psi_{t} \psi_{t}^{\star}\right)^{-1}=U_{t}^{\star}\left(\bar{a}\left(Y_{t}\right) \bar{a}^{\star}\left(Y_{t}\right)\right)^{-1} U_{t} \leq C U_{t}^{\star} U_{t}
$$

SO

$$
\psi_{t} \psi_{t}^{\star} \geq V_{t} I
$$

for a positive variable $V_{t}$ satisfying

$$
\left\|\sup _{t>\sigma_{k}} V_{t}^{-1}\right\|_{p}<\infty
$$

Thus, for any $k$,

$$
\begin{aligned}
\left(\int_{0}^{T} \psi_{t} \psi_{t}^{\star} d t\right)^{-1} & \leq I\left(\int_{\sigma_{k}}^{T} V_{t} d t\right)^{-1} \\
& \leq I\left(T-\sigma_{k}\right)^{-1} \sup _{t>\sigma_{k}} V_{t}^{-1} .
\end{aligned}
$$

The variable $T-\sigma_{k}$ is the sum (limited to $T$ ) of $k$ independent exponential variables, so its inverse is in $L^{p}$ if $k$ is chosen large enough, and we can conclude.

Remark 1. If one assumes that the left hand side of (4.16) is bounded below by a positive constant, the proof of the corollary can be shortened by noticing that $\left(Z_{T}^{t}\right)^{-1}$ has bounded moments, so that $\left(\psi_{t} \psi_{t}^{\star}\right)^{-1}$ also has bounded moments.

Remark 2. Our assumption (4.16) deals with the behaviour at big jumps; it implies that the semi-flow $\phi_{s t}$ is locally injective, but not necessarily globally, as it can be seen in the two-dimensional example

$$
a(y, x)=x \quad \text { if } \quad x \neq x_{0}
$$




$$
a\left(y, x_{0}\right)=\left(2+\arctan y_{1}\right)\left(\begin{array}{c}
\cos y_{2} \\
\sin y_{2}
\end{array}\right)-y
$$

when $\mu\left(\left\{x_{0}\right\}\right)>0$.

Remark 3. If the Lévy measure $\mu$ does not charge similarly all the directions, one can try to proceed as explained in the end of $\S 3$. However, some results which were previously obtained with the classical Malliavin calculus do not follow directly from our results, and really need a precise study of noises with different levels such as [6].

Remark 4. In contrast with the continuous case, the ellipticity condition for $\bar{a} \bar{a}^{\star}$ must hold everywhere and not only at the origin $y_{0}$; a big jump can indeed make the solution to exit the ellipticity domain too quickly, so that the density cannot be bounded. Note also that the ellipticity condition without the local invertibility of the flow is not sufficient.

We now consider an example which satisfies a Hörmander condition rather than the ellipticity condition of Corollary 4.4; this will be the classical Lévy stochastic area considered in the case of Lévy processes.

Corollary 4.5. Let $X_{t}=\left(X_{t}^{1}, X_{t}^{2}\right)$ be a two-dimensional Lévy process satisfying the assumptions of Corollary 1.2(b), and such that

$$
\int|x|^{p} d \mu(x)<\infty
$$

for any $p \geq 2$. Define

$$
L_{T}=\frac{1}{2}\left(\int_{0}^{T} X_{t-}^{1} d X_{t}^{2}-\int_{0}^{T} X_{t-}^{2} d X_{t}^{1}\right) .
$$

Then $Y_{T}=\left(X_{T}, L_{T}\right)$ has a $C_{b}^{\infty}$ density for any $T>0$.

Proof. This case corresponds to the equation (4.1) with coefficients

$$
b=\widetilde{a}=0, \quad \bar{a}(y)=\left(\begin{array}{cc}
1 & 0 \\
0 & 1 \\
-y_{2} / 2 & y_{1} / 2
\end{array}\right) .
$$

After some computation, one obtains

$$
\begin{aligned}
\operatorname{det} \int_{0}^{T} \psi_{t} \psi_{t}^{\star} d t & =T^{2} \int_{0}^{T}\left|X_{t}-\frac{1}{T} \int_{0}^{T} X_{s} d s\right|^{2} d t \\
& \geq T^{2} \sum_{j=0}^{k-1} \int_{t_{j}}^{t_{j+1}}\left|X_{t}-\frac{k}{T} \int_{t_{j}}^{t_{j+1}} X_{s} d s\right|^{2} d t \\
& =\frac{k T}{2} \sum_{j=0}^{k-1} \int_{t_{j}}^{t_{j+1}} \int_{t_{j}}^{t_{j+1}}\left|X_{t}-X_{s}\right|^{2} d s d t
\end{aligned}
$$


for the regular subdivision $t_{j}=j T / k$ of $[0, T]$. For any $\delta>0$, the variables $X_{t}-X_{s}$, $|t-s| \geq \delta$, have uniformly bounded densities, so

$$
\sup \left\{\mathbb{E}\left[\left|X_{t}-X_{s}\right|^{-1}\right] ;|t-s| \geq \delta\right\}<\infty,
$$

and therefore

$$
\mathbb{E}\left(\int_{t_{j}}^{t_{j+1}} \int_{t_{j}}^{t_{j+1}}\left|X_{t}-X_{s}\right|^{2} d s d t\right)^{-1 / 2}<\infty
$$

The variables corresponding to different values of $j$ are independent, so

$$
\mathbb{P}\left[\operatorname{det} \int_{0}^{1} \psi_{t} \psi_{t}^{\star} \leq \eta\right] \leq \prod_{j=0}^{k-1} \mathbb{P}\left[\int_{t_{j}}^{t_{j+1}} \int_{t_{j}}^{t_{j+1}}\left(X_{t}^{1}-X_{s}^{1}\right)^{2} d s d t \leq 2 \eta /(k T)\right] \leq C_{k} \eta^{k / 2}
$$

so the inverse of the determinant is proved to have a finite $p$ th moment by choosing $k$ large enough.

\section{References}

[1] K. Bichteler, J.B. Gravereaux and J. Jacod, Malliavin calculus for processes with jumps, Stochastics Monographs 2, Gordon and Breach, 1987.

[2] J.M. Bismut, Calcul des variations stochastique et processus de sauts, Z. Wahrscheinlichkeitstheorie verw. Gebiete 63 (1983), 147-235.

[3] E. Carlen and E. Pardoux, Differential calculus and integration by parts on Poisson space, in: Stochastics, Algebra and Analysis in Classical and Quantum Dynamics (Marseille, 1988), 63-73, Math. Appl. 59, Kluwer, 1990.

[4] T. Fujiwara and H. Kunita, Stochastic differential equations of jump type and Lévy processes in diffeomorphisms group, J. Math. Kyoto Univ. 25 (1985), 1, 71-106.

[5] R. Léandre, Flot d'une équation différentielle stochastique avec semi-martingale directrice discontinue, in: Séminaire de Probabilités XIX, 271-274, Lect. N. Math. 1123, Springer, 1985.

[6] R. Léandre, Régularité de processus de sauts dégénérés, Ann. Inst. Henri Poincaré, Prob. et Stat. 21 (1985), 2, 125-146.

[7] R. Léandre, Régularité de processus de sauts dégénérés (II), Ann. Inst. Henri Poincaré, Prob. et Stat. 24 (1988), 209-236.

[8] P. Malliavin, Stochastic calculus of variation and hypoelliptic operators, in: Proc. Intern. Symp. SDE (Kyoto, 1976), 195-263, Wiley, 1978.

[9] P.A. Meyer, Flot d'une équation différentielle stochastique, in: Séminaire de Probabilités XV, 103-117, Lect. N. Math. 850, Springer, 1981.

[10] D. Nualart and J. Vives, Anticipative calculus for the Poisson process based on the Fock space, in: Séminaire de Probabilités XXIV, 154-165, Lect. N. Math. 1426, Springer, 1990.

[11] D. Nualart and J. Vives, A duality formula on the Poisson space and some applications, in: Seminar on Stochastic Analysis, Random fields and Applications (Ascona, 1993), Progress in Probability 36, Birkhäuser, 1995. 
[12] J. Picard, Formules de dualité sur l'espace de Poisson, Ann. Inst. Henri Poincaré, Prob. et Stat., to appear.

[13] K. Sato, Absolute continuity of multivariate distributions of class L, J. Multivariate Anal. 12 (1982), 1, 89-94.

[14] H.G. Tucker, On a necessary and sufficient condition that an infinitely divisible distribution be absolutely continuous, Trans. American Math. Soc. 118 (1965), 316-330. 